\title{
Exploring inhibition of Pdx1, a component of the PLP synthase complex of the human malaria parasite Plasmodium falciparum
}

Shaun B. Reeksting*†, Ingrid B. Müller†, Pieter B. Burger*, Emmanuel S. Burgos ${ }^{\dagger}$, Laurent Salmon§, Abraham I. Louw*, Lyn-Marie Birkholtz* and Carsten Wrenger $\dagger \|^{1}$

* Department of Biochemistry, Faculty of Natural and Agricultural Sciences, University of Pretoria, Private Bag x20, Hatfield 0028, South Africa

$\dagger$ Bernhard Nocht Institute for Tropical Medicine, Department of Biochemistry, Bernhard Nocht-Straße 74, D-20359, Germany

\$ Albert Einstein College of Medicine, Biochemistry Department, 1300 Morris Park Avenue, Bronx, NY 10461, U.S.A.

$\S$ Laboratoire de Chimie Bioorganique et Bioinorganique, CNRS-UMR 8182, Institut de Chimie Moléculaire et des Matériaux d'Orsay, Bâtiment 420, Univ Paris Sud XI, F-91405 Orsay, France

|| Department of Parasitology, Institute of Biomedical Science, University of São Paulo, Av. Prof. Lineu Prestes 1374, 05508-000 São Paulo-SP, Brazil

${ }^{1}$ To whom correspondance should be addressed (Phone: +5511 3091 7335, Email: cwrenger@icb.usp.br).

Short title: Inhibition of P. falciparum PLP synthase

Keywords: Pyridoxal 5-phosphate (PLP), vitamin $\mathrm{B}_{6}$, PLP synthase, Pdx1, inhibition, erythrose 4-phosphate (E4P).

Abbreviations: 4PEHz, 4-phospho-D-erythronhydrazide; ANCOVA, analysis of covariance; DHAP, dihydroxyacetone phosphate; DR5P, 2-deoxy-D-ribose 5 phosphate; DXP, 1-deoxy-Dxylulose 5-phosphate; E4P, D-erythrose 4-phosphate; EHz, D-erythronhydrazide; G3P, DLglyceraldehyde 3-phosphate; PLP, pyridoxal 5'-phosphate; PNP, pyridoxine 5'-phosphate; R5P, D-ribose 5-phosphate; Ru5P, D-ribulose 5-phosphate; RPI, R5P isomerase; RMS, root mean square; RMSD, root mean square deviation; TIM, triose phosphate isomerase. 


\section{Synopsis}

Malaria tropica is a devastating infectious disease caused by Plasmodium falciparum. This parasite synthesizes vitamin $\mathrm{B}_{6}$ de novo via the pyridoxal 5-phosphate (PLP) synthase enzymatic complex consisting of PfPdx1 and PfPdx2 proteins. Biosynthesis of PLP is largely performed by $P f \mathrm{Pdx} 1$, ammonia provided by $P f \mathrm{Pdx} 2$ subunits, is condensed together with Dribose 5-phosphate (R5P) and DL-glyceraldehyde 3-phosphate (G3P). PfPdx1 accommodates both the R5P and G3P substrates and intricately coordinates the reaction mechanism, which is composed of a series of imine bond formations, leading to the production of PLP. We demonstrate that D-erythrose 4-phosphate (E4P) inhibits PfPdx1 in a dose dependent manner. We propose that the acyclic phospho-sugar E4P, with a $\mathrm{C} 1$ aldehyde group similar to acyclic R5P, could interfere with R5P imine bond formations in the PfPdx1 reaction mechanism. Molecular docking and subsequent screening identified the E4P hydrazide analogue, 4phospho-D-erythronhydrazide (4PEHz), which selectively inhibited PfPdx 1 with an $\mathrm{IC}_{50}$ of 43 $\mu \mathrm{M}$. PfPdx1 contained in the heteromeric PLP synthase complex was shown to be more sensitive to $4 \mathrm{PEHz}$ and was inhibited with an $\mathrm{IC}_{50}$ of $16 \mu \mathrm{M}$. Moreover, the compound had an $\mathrm{IC}_{50}$ value of $10 \mu \mathrm{M}$ against cultured $P$. falciparum intraerythrocytic parasites. To further analyse the selectivity of $4 \mathrm{PEHz}$, transgenic cell lines over-expressing PfPdx1 and PfPdx2 showed that additional copies of the protein complex conferred protection against $4 \mathrm{PEHz}$, implicating that the PLP synthase is directly affected by $4 \mathrm{EPHz}$ in vivo. These PfPdx1 inhibitors represent novel lead scaffolds which are capable of targeting PLP biosynthesis, and we propose this as a viable strategy for the development of newer therapeutics against malaria.

\section{Introduction}

The parasitic disease, malaria, caused by members of the genus Plasmodium, represents one of the most serious threats to humans worldwide. More than 2 billion people are exposed to the parasites leading to an estimated 500 million clinical cases and more than 1 million deaths per annum, mostly young children in Africa. An effective vaccine is not available and prophylaxis as well as chemotherapy depends solely on the administration of a small number of drugs $[1,2]$. There are less than 30 different small molecule scaffolds or chemotypes that are effective against $P$. falciparum parasites, and the clinically-employed repertoire of antimalarials consists of only around 10 different scaffolds [3]. At the current rate of parasite drug-resistance development this handful of antimalarials are under threat of rapidly becoming ineffective. For this reason the discovery of newer chemical scaffolds and the exploitation of novel drug targets in this parasite are of utmost importance.

PLP is the catalytic active form of vitamin $\mathrm{B}_{6}$ and a vital prosthetic group to a wide range of enzymes primarily involving amino acid biosynthesis [4]. The cofactor is extremely versatile, and partakes in enzyme-catalysed racemizations, transaminations, decarboxylations and substitution reactions [4]. During catalysis PLP functions to stabilize the negative charge at the $\mathrm{C}_{\alpha}$ of a PLP-amino acid intermediate [4]. Recently, it became apparent that the function of PLP in P. falciparum parasites is not only restricted to its action as a prosthetic group. The molecule is required in response towards oxidative stress caused by the generation of singlet oxygen during hemoglobin digestion [5, 6]. Currently two known PLP-biosynthetic pathways exist; the 1-deoxy-D-xylulose 5-phosphate (DXP)-dependent pathway and the DXPindependent route. The DXP-dependent pathways is present in Escherichia coli and some proteobacteria [7]. The pathway involves several enzymes which condense D-erythrose 4phosphate (E4P), DXP and glutamate to form pyridoxine 5'-phosphate (PNP), which is subsequently converted to PLP [8]. The DXP-independent pathway, consisting of the enzymes $\operatorname{Pdx} 1$ and $\mathrm{Pdx} 2$, is found in plants, fungi as well as the human pathogens 
Plasmodium falciparum, Toxoplasma gondii and Mycobacterium tuberculosis [5, 9]. The pathway is independent of DXP and instead R5P, G3P and L-glutamine are utilised to form PLP. P. falciparum parasites have a functional DXP-independent pathway consisting of a PLP synthase, which is made up of PfPdx 1 and $P f P d x 2$. Human hosts of the malaria parasite lack the biosynthetic enzymes and are unable to produce PLP, making this parasite-exclusive pathway an attractive target for the development of therapeutics.

The catalytic centre of the PLP synthase is Pdx1, where R5P, G3P and ammonia is combined through an intricately complex biochemical process [10]. Pdx1 proteins have been shown to alternatively utilise D-ribulose 5-phosphate (Ru5P) and dihydroxyacetone phosphate (DHAP) as substitute pentose and triose substrates, respectively [11-13]. The protein is therefore thought to contain R5P isomerase (RPI) and triose phosphate isomerase (TIM) activities in order to convert these alternative substrates. The reaction mechanism of PLP formation in $\mathrm{Pdx} 1$ has been extensively elucidated. The reaction in Pdx1 is initiated by R5P ring opening and the formation of an imine adduct between R5P and an internal K83 residue [10]. Initial binding of R5P to the internal $\mathrm{K}$ residue occurs via the $\mathrm{C} 1$ aldehyde group [10, 14]. Binding of R5P is followed by isomerization into an Ru5P intermediate, which eventually leads to the formation of an internal chromophore with characteristic absorbance at $320 \mathrm{~nm}$, termed $\mathrm{I}_{320}$ $[10,14,15]$. The addition of G3P to Pdx1 with bound $\mathrm{I}_{320}$ forms leads to the formation and release of PLP [10]. It is thought that the furanose form of R5P is bound by Pdx1, however the exact mechanism of R5P ring opening remains unclear [15].

Here, we report the identification of PfPdx1 inhibitors capable of interfering with PLP production. Analogues of R5P and G3P, in which stereo chemical arrangement and hydrolyzable phosphate groups were retained, were found to inhibit PfPdx1 more effectively than their non-phosphorylated counterparts. The $\mathrm{C} 1$ aldehyde reactivity found on some of these inhibitors was also an important feature. Our studies revealed inhibitors with appreciable inhibitory effect against $P$. falciparum parasites in vitro and we demonstrated target specificity against PfPdx1 in vivo.

\section{Experimental}

\section{Materials}

D-Erythronhydrazide (EHz) was synthesized by iThemba pharmaceuticals (South Africa) and supplied as HPLC purified product, which was structurally confirmed by ${ }^{1} \mathrm{H}-\mathrm{NMR}$ and mass spectrometry. All other chemical compounds including R5P, G3P, E4P and D-erythrose were purchased from Sigma Aldrich unless otherwise stated. Albumax II and gentamycin were obtained from Invitrogen. $\left[{ }^{3} \mathrm{H}\right]$-hypoxanthine (specific activity $30 \mathrm{Ci} / \mathrm{mmol} ; 0.03 \mathrm{mM}$ ) was purchased from American Radiolabeled Chemicals and Ultima Gold scintillation fluid was from Perkin-Elmer. Restriction enzymes and ligases were purchased from New England Biolabs, USA. Oligonucleotides were obtained from Sigma Aldrich. The cloning vector pASK-IBA3, Strep-Tactin-Sepharose, anhydrotetracycline (AHT) and desthiobiotin were obtained from the Institut für Bioanalytik (IBA), Germany.

\section{In silico modelling and ligand screening}

Molecular modelling was performed using the Discovery Studio suite (v3.0, Accelrys Software Inc.) unless specified otherwise. PfPdxl homology models were generated using $T$. maritima (TmPdx1, PDB accession: 2ISS) and Thermus thermophilus (TtPdx1, PDB accession: 2ZBT) Pdx 1 as templates. At the time this study was initiated the crystal structure of P. berghei Pdx1 (PbPdx1, PDB accession: 4ADU) [16] was not available. Several 
homology models were created, each incorporating a different ligand from the template structures, including Ru5P and 2-methyl-2,4-pentanediol, as well as surrounding water molecules. The quality of the structures was assessed using model-derived pseudo-energy terms as well as stereochemical pseudo-energy terms and Ramachandran plots were generated in PDBsum $[17,18]$. Analysis with PROCHECK showed a slight decrease in the average G factors between the crystal structure (0.39-0.41) and the homology models $(0,00)$; scores above -0.5 are considered acceptable $[17,18]$. The homology models were subjected to 200 steps of steepest descent energy minimization using the CHARMm forcefield with a root mean square (RMS) gradient tolerance of 0.1 , followed by 200 steps conjugate gradient minimization. R5P was selected from the optimized protein-substrate complex to define a binding cavity and assigned a sphere radius of $8 \AA$. The defined binding cavity was used to extract pharmacophore features from the different PfPdx1 models, followed by clustering and high throughput virtual screening of 2 million compounds of the Zinc database drug-like subset [19]. The database was generated as a multi-conformer composite database using catDB with a maximum of 250 representative conformations (Accelrys Software Inc.). The drug-like database was screened against 5 pharmacophore models and 300 best-hit compounds were identified based on their pharmacophore fit-values. These compounds were subjected to molecular docking simulations using LigandFit employing iterative rigid body minimization in a dreiding energy forcefield with 1500 Monte Carlo trials [20, 21]. The ligand poses were ranked according to the docking score (DockScore). In addition ligand poses were evaluated using LigScore empirical scoring functions [22]. R5P was used as control in cross-docking to validate ligand docking and scoring. Graphical displays were generated with VMD [23].

Following the release of the $P b P d x 1$ structure in 2012 [16], additional docking was performed on the $P f \mathrm{Pdx} 1$ homolog. The $P b \mathrm{Pdx} 1$ structure was prepared by removing the covalent bond between K84 and R5P, followed by minimization as described above. Post-minimization, R5P was selected to assign a binding site and sphere radius, similar to the docking simulations described above. K84 was maintained in a protonated state during the docking simulations (predicted $\mathrm{pK}_{\mathrm{a}}$ of 12.1). Ligands were docked and scored using LigandFit and LigScore, as described above.

\section{Mutagenesis of PfPdxl}

Deletion of the C-terminal region of PfPdx1 was performed on PfPdx1 previously cloned in the expression plasmid pASK-IBA3 (IBA, Germany) [5]. Multiple deletion mutagenesis PCR's were set-up, and contained 35 ng of expression plasmid with 30 pmol each of the sense primer (5'-GCGCGCGGTCTCGAATGGAAAATCATAAAGATGATGC-3') and antisense primer (5'-GCGCGCGGTCTCAGCG*CTAACATCTAAAAGTATTTTAGGG-3') (site of Cterminal truncated region indicated with asterisk) in a final volume of $50 \mu \mathrm{L}$ with PCR Supermix (Invitrogen) containing recombinant Thermus aquaticus DNA polymerase. The cycling parameters were $95{ }^{\circ} \mathrm{C}$ for $3 \mathrm{~min}$, then 35 cycles of $95{ }^{\circ} \mathrm{C}$ for $30 \mathrm{~s}, 41^{\circ} \mathrm{C}$ for $90 \mathrm{~s}, 60$ ${ }^{\circ} \mathrm{C}$ for $2 \mathrm{~min}$. Parent template was removed from the reactions by incubation with $20 \mathrm{U}$ of DpnI (New England Biolabs) for $2 \mathrm{~h}$ at $37^{\circ} \mathrm{C}$. Following Invitrogen Pure link PCR purification $50 \mu \mathrm{L}$ eluted solutions were digested with $10 \mathrm{U}$ of $B s a \mathrm{I}$ (New England Biolabs) for $3 \mathrm{~h}$ at $37^{\circ} \mathrm{C}$, purified, and ligated $(16 \mu \mathrm{L})$ into similarly digested pASK-IBA3 expression plasmids at a ratio of 3:1 insert to vector using T4-DNA ligase (New England Biolabs). Ligation reactions $(20 \mu \mathrm{L})$ were transformed directly into $200 \mu \mathrm{L}$ XL10 Gold competent cells (Stratagene) using heat shock transformation for $60 \mathrm{~s}$ at $42^{\circ} \mathrm{C}$, cooling on ice, followed by the addition of $800 \mu \mathrm{L}$ Luria-Bertani (LB) medium. Cells were plated onto LB agar plates containing $50 \mu \mathrm{g} / \mathrm{mL}$ ampicillin (LB-amp) and grown for $16 \mathrm{~h}$ at $37^{\circ} \mathrm{C}$. Single colonies were selected and used to inoculate $2 \mathrm{~mL}$ LB-amp medium, and grown for $16 \mathrm{~h}$ at $37^{\circ} \mathrm{C}$. Plasmids were extracted using the peqGold plasmid miniprep kit I (Peqlab). Recombinant plasmids (10 
$\mu \mathrm{L}$ ) were confirmed through $\mathrm{Xba \textrm {I }}(5 \mathrm{U})$ and Hind III (5U) (New England Biolabs) restriction enzyme digestions and automated sequencing (Seqlab, Germany). The PfPdx1 R164A mutation was created with sense primer 5'GCTATTAAACATATAGCAACTGTAAATAATGAA-3' and antisense primer 5'TTCATTATTTACAGTTGCTATATGTTTAATAGC-3' (mutated residues indicated in bold and underlined) as described previously [24].

\section{Expression and purification of PfPdx1 and PfPdx2}

PfPdx1 and PfPdx2 clones in pASK-IBA3 were transformed into E. coli BLR (DE3) [5]. Selected single colonies were used to inoculate LB medium containing $50 \mu \mathrm{g} / \mathrm{mL}$ ampicillin (LB-amp) and grown overnight at $37^{\circ} \mathrm{C}$. Cultures were diluted 1:100 into LB-amp media and grown to an $\mathrm{OD}_{600}$ of 0.5. Expression was induced using $200 \mathrm{ng} / \mathrm{mL}$ AHT (IBA) and grown for $4 \mathrm{~h}$ at $37{ }^{\circ} \mathrm{C}$ with agitation. Cells were collected by centrifugation at $2000 \mathrm{~g}$ for $10 \mathrm{~min}$ and the pellets were resuspended in $100 \mathrm{mM}$ Tris- $\mathrm{HCl}$ and $150 \mathrm{mM} \mathrm{NaCl}$ at $\mathrm{pH} 8.0$. Phenylmethylsulfonyl fluoride $(0.1 \mathrm{mM})$ and lysozyme $(5 \mathrm{mg} / 10 \mathrm{~mL}$ reconstituted pellet $)$ was added, the pellets sonicated using pulsing at output 6 , duty cycle 40 with 1 min resting intervals on ice (Branson Sonifier 250) and clarified by centrifugation at $50000 \mathrm{~g}$ for $50 \mathrm{~min}$ at $4{ }^{\circ} \mathrm{C}$. Strep-Tag fusion proteins were purified according to manufacturer's recommendations (IBA).

\section{Enzyme activity measurements}

PfPdx1 assays were performed using the $100 \mathrm{mM}$ Tris-Cl and $150 \mathrm{mM} \mathrm{NaCl}$ buffer $(\mathrm{pH} 8.0)$ as previously described [25]. The PfPdx1 assay contained $0.5 \mathrm{mM} \mathrm{R} 5 \mathrm{P}, 0.5 \mathrm{mM}$ G3P and 20 $\mathrm{mM} \mathrm{NH} \mathrm{NH}_{4} \mathrm{Cl}$ in a final assay volume of $250 \mu \mathrm{L}$ with $200 \mu \mathrm{g}(6 \mathrm{nM})$ of purified PfPdx1. Initially compounds were only screened against PfPdx1 to discount molecules that could have interfered with $P f \mathrm{Pdx} 1$ and $P f \mathrm{Pdx} 2$ heterodimerization. In assays containing both $P f \mathrm{Pdx} 1$ and PfPdx2 $20 \mathrm{mM}$ L-glutamine was used instead of $\mathrm{NH}_{4} \mathrm{Cl}$. Reactions used for baseline normalization included purified PfPdx1 protein, G3P and $\mathrm{NH}_{4} \mathrm{Cl}$, without R5P. Reactions were incubated at $37^{\circ} \mathrm{C}$ for $2.5 \mathrm{~h}$ and the PLP-Tris Schiff-base was detected at $414 \mathrm{~nm}$. Spectrophotometric measurements, including wavelength scans, were performed with a Uvikon 933B spectrophotometer (Bio-Tek Kontron) in $70 \mu \mathrm{L}$ cuvettes (Plastibrand, Brand) or using a Nanodrop 1000 (ND).

\section{Culturing of $P$. falciparum and $I C_{50}$ assays}

P. falciparum (3D7) parasites were maintained in continuous culture according to Trager et al. [26], as modified by Das Gupta et al. [27]. The RPMI 1640 culture medium contained 25 $\mathrm{mM}$ HEPES, $10 \mathrm{mM}$ glucose, $20 \mathrm{mM}$ sodium bicarbonate, $25 \mathrm{mM}$ hypoxanthine and $0.5 \%$ w/v AlbuMAX II (Invitrogen) at pH 7.4. The RPMI 1640 media as supplied by Applichem contains $0.001 \mathrm{~g} / \mathrm{L}$ or $4.86 \mu \mathrm{M}$ pyridoxine hydrochloride. Parasite cultures were maintained at $4 \%$ hematocrit using human $\mathrm{O}+$ erythrocytes in flasks (Nunc) with shaking at $200 \mathrm{rpm}$ at $37^{\circ} \mathrm{C}$. Parasite cultures $(50 \mathrm{~mL})$ consisting of ring stage parasites ( 1 to $5 \%$ parasitemia) were

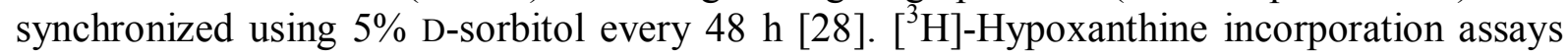
were performed as described elsewhere [27] on ring stage parasites $(2 \%$ hematocrit and $1 \%$ parasitemia) exposed to serial dilutions of test compound in 96 well plates at $37^{\circ} \mathrm{C}$. After 24 $\mathrm{h}, 100 \mathrm{nCi}\left[{ }^{3} \mathrm{H}\right]$-hypoxanthine was added and parasites cultures were incubated for an additional $24 \mathrm{~h}$, followed by cell harvesting (Inotech) and collection on filter mats. Filter mats were washed four times using distilled water, dried at $60^{\circ} \mathrm{C}$ for $10 \mathrm{~min}$ and suspended in $4 \mathrm{~mL}$ Ultima Gold ${ }^{\mathrm{TM}}$ scintillation fluid (Perkin Elmer) and counted using a Trilux liquid scintillation counter (Wallace). The $50 \%$ inhibitory concentrations $\left(\mathrm{IC}_{50}\right)$ were calculated from sigmoid dose response curves generated using GraphPad Prism 5 (GraphPad Software Inc.). 


\section{Long-term growth assays}

Asynchronous $P$. falciparum parasites, co-transfected with PfPdx1 and PfPdx2 pARL1a vectors with blasticidin S and WR99210 antibiotic resistance, together with a mock cell line, containing vector with only antibiotic resistance, were maintained in culture, as described previously [6]. Blasticidin S-hydrochloride (Sigma Aldrich) and WR99210 (Jacobus Pharmaceuticals) were maintained at final concentrations of $2.1 \mathrm{mM}$ and $5 \mathrm{nM}$, respectively. Long-term growth assays were performed, as previously described [29], using 12 well plates (Nunc) at 4\% hematocrit, with 1\% starting parasitemia. Both PfPdx1/PfPdx2-overexpressing and mock parasites were maintained in culture for 7 days. Spent media was removed from suspended erythrocytes by gently tilting the plates, and replaced with fresh media containing both antibiotics. Fresh $4 \mathrm{PEHz}$ was added to both treated-PfPdx1/PfPdx2 as well as treatedmock parasites every day at a final concentration of $1 \mu \mathrm{M}$. The growth rate was assessed by morphological monitoring and counting of Giemsa-stained thin smears using oil immersion light microscopy at $100 x$ magnification, counting $\geq 1000$ red blood cells from each slide. The cumulative parasitemia was calculated using the determined parasitemia by factoring in the dilution. From the log transformed data the slope of the regression line was used to calculate the growth rate constant $(\mathrm{K})$, by using the following equation;

$$
K=\ln \left(10^{\text {slope }}\right)
$$

Results were analysed using GraphPad Prism 5 (GraphPad Software Inc.), in which the null hypothesis of equal regression lines slopes was tested at a $99 \%$ confidence interval $(P=0.01)$. Analysis of covariance (ANCOVA) was conducted (JMP, Version 9, SAS Institute Inc.), testing whether the slopes significantly differed from each other with $\alpha=0.05$.

\section{Results}

\section{In silico docking and screening of PfPdxl inhibitors}

Homology models of PfPdx1 were generated from T. maritima (TmPdx1) and T. thermophilus (TtPdx1) Pdx1 templates that share a $60 \%$ and $61 \%$ sequence identity with PfPdx1, respectively. The resulting models showed an average RMSD of $3.7 \AA$ and $2.4 \AA$ compared to the $\mathrm{C}_{\alpha}$ backbone of the $T m \mathrm{Pdx} 1$ and $T t \mathrm{Pdx} 1$ templates, respectively. Comparing the active site residues (residues within $5 \AA$ of the substrates) revealed a high degree of structural conservation with RMSD values of $0.7 \AA$ and $1.0 \AA$, respectively. Moreover, a structural comparison of our models to the recently released P. berghei $\mathrm{Pdx} 1$ (PbPdx1), which shares a $85 \%$ sequence identity with $P f \mathrm{Pdx} 1$, showed a highly conserved $\mathrm{C}_{\alpha}$ backbone RMSD ranging between $1.2 \AA$ and 1.3 $\AA$. Moreover, the active site residues of the PfPdx1 models showed RMSD values of between $0.6 \AA$ and $0.7 \AA$, confirming the quality and validity of the models. Five diverse pharmacophore models descriptive of the PfPdx1 R5P binding site were derived from the protein models and screened against the drug-like subset of the Zinc database. Using the 300 best-fitting compounds, additional molecular docking was used to refine and determine binding capabilities in the R5P active site (Table 1). Following docking, a total of 19 compounds were selected for in vitro testing against purified PfPdx1 whereby PLP formation was monitored at a wavelength of $414 \mathrm{~nm}$ according to Wrenger et al. [5], (Table 1). Compounds 1-4 contained sulfonate moieties and showed similar docking scores to that of R5P, however had no appreciable activity against PfPdxl at $3 \mathrm{mM}$ (Table 1). Four polyhydroxylated compounds were identified from the PfPdx1 pharamacophores (compounds 5-8). Compound 8 which had a terminal keto-ester arrangement was the most effective of the 
polyhydroxylated compounds tested, with a $12 \%$ reduction of PfPdx1 activity at $0.5 \mathrm{mM}$, although not statistically significant (Table 1). Compounds 9, 13 and 16, which share a pyrimidine sulfonamide moiety, were not effective at inhibiting PfPdx1 (Table 1). Fosmidomycin (compound 17), which was predicted to have a high docking score, had no inhibitory effect on PfPdx1 activity at $20 \mathrm{mM}$ (Table 1).

We investigated whether molecules closely related to the PfPdx1 substrate R5P were capable of inhibiting the protein. The R5P analogues, 2-deoxy-D-ribose 5 phosphate (DR5P, compound 18) and 1-deoxy-D-xylulose 5-phosphate (DXP, compound 19), had similar docking scores compared to R5P (Table 1). This suggested that DR5P could have favourable binding interactions in the R5P-binding site. When tested, DR5P was a weak inhibitor of PfPdx 1 even at concentrations as high as $12 \mathrm{mM}$ with $56 \%(P<0.10)$ of the enzyme activity remaining (Figure 1A). DXP decreased PfPdx1 activity to $88 \%$ at $5 \mathrm{mM}$ (Table 1). Considering that initial binding of R5P to Pdx1 entails imine formation with K83, DR5P could compete for this interaction [15]. Spectrophotometric UV-visible absorbance spectra of PfPdx1 incubated with DR5P revealed minor decreases in $\mathrm{I}_{320}$ formation with increasing concentrations of DR5P (Figure 1A). Decreased PLP production suggested that the molecule could potentially interfere with R5P-K83 imine adduct formation and/or PLP formation.

The specificity and stringency of PfPdx1 for other R5P analogues that could be either incorporated into the PLP ring structure and/or interfere with R5P imine adduct formation, was tested next. Several sugars including E4P, F6P, DR5P (Figure 1B) and DXP (results not shown) did not support the formation of PLP, confirming the strict substrate specificity of PfPdx1. Interestingly we noted that substitution of R5P by E4P resulted in the formation of the single $\mathrm{I}_{320}$ chromophore similar to PfPdx1 incubated with R5P in the absence of G3P (Figure 1B). The structural resemblance between E4P and R5P suggests that the former could compete with R5P for Schiff base formation with the internal K83 residue. Molecular docking simulations predicted similar LigScores for E4P and R5P in PfPdx1 models (Table 2). Similarly, docking of E4P into the PbPdx1 was comparable to the R5P substrate (Figure 1C, Table 2). E4P was orientated with the aldehyde group in close proximity to the K84 residue (Figure 1D). These predicted interactions were investigated in more detail below.

\section{Intensive analysis of E4P as PfPdxl inhibitor}

The activity of PfPdx1 was assayed in the presence of varying concentrations of E4P, and a dose-dependent decline in PLP synthesis was observed (Figure 2A). A concomitant increase in absorbance at $320 \mathrm{~nm}$ was observed for increasing concentrations of E4P (Figure 2A). To discard potential conjugation or adduct formation of PLP by E4P, calibration curves of increasing concentrations of PLP were used to show that $1 \mathrm{mM}$ E4P did not significantly affect detection of PLP at $414 \mathrm{~nm}(\mathrm{n}=4, P=0.975$, Suppl. Figure 1). This confirmed that E4P was responsible for inhibiting the production of PLP by PfPdx1. E4P inhibited the formation of PLP by PfPdx 1 with an $\mathrm{IC}_{50}$ value of $3.7 \pm 0.9 \mathrm{mM}$ (Figure $2 \mathrm{~B}$, Table 2). The unphosphorylated analogue of E4P - D-erythrose - similarly interfered with PLP formation but was more than 40 times weaker compared to E4P (Figure 2B, Table 2). This emphasized the role of such hydrolyzable phosphate groups with the additional negative charge potentially facilitating entry into the PfPdx1 active site.

\section{Residues involved in $I_{320}$ formation}

Recent studies have revealed several important residues involved in $\mathrm{I}_{320}$ formation and include D26, K83 and K151 [24] as well as residues from the C-terminus [30]. We sought to verify the importance of these residues during PfPdx1 $\mathrm{I}_{320}$ formation. Incubation of R5P with both WT Pdx1 and ERR (E136A, R139A and R140A) PfPdx1 resulted in $\mathrm{I}_{320}$ formation, however, the DKK (D26A, K83A and K151A) mutant was unable to form this species (Figure 3A). 
This corroborates previous findings and excludes the involvement of the ERR residues located in the PLP binding site in $\mathrm{I}_{320}$ formation [31]. A RHE (R85A, H88A and E91A) PfPdxl mutant was previously shown to prevent dodecameric assembly, and to be incapable of activating PfPdx2 subunits [24]. These residues are therefore believed to coordinate Pdx1$\mathrm{Pdx} 1$ subunit cross talk. Additional experimentation with RHE PfPdx1 mutants and $\triangle \mathrm{C} \mathrm{Pdx1,}$ lacking the $\pm 30 \mathrm{C}$-terminal residues, revealed that both proteins were unable to form $\mathrm{I}_{320}$ (Figure 3B). These observations are in agreement with functions assigned to the C-terminal region and moreover emphasized the importance of the RHE residues for R5P binding and imine adduct formation [30]. Reduced formation of $\mathrm{I}_{320}$ in R167A PfPdx1 suggested that this residue, which is part of the G3P binding site, indirectly influences the way $\mathrm{R} 5 \mathrm{P}$ is processed (Figure 3C). Contrary to observations elsewhere, this mutation decreased enzyme activity by only $\sim 50 \%$ (Figure $3 \mathrm{C}$ ) [13].

\section{E4P analogues show inhibitory activity against PfPdxI}

From the E4P lead structure a closely related hydrazide analogue $4 \mathrm{PEHz}$ [32], and the unphosphorylated form of $4 \mathrm{PEHz}$, termed $\mathrm{EHz}$, were selected and tested against PfPdxl. Previously, 4PEHz was shown to have weak inhibitory activity against spinach RPI [32]. Molecular docking simulations predicted favourable ligand poses for $4 \mathrm{PEHz}$ in the R5P cavity of PfPdx1, as well as in PbPdx1 (Table 2). The EHz molecule was predicted to have less favourable binding interactions in PfPdx1 and had smaller Ligscore binding affinities compared to $4 \mathrm{PEHz}$ (Table 2). 4PEHz could be docked into the PbPdx1 and had hydrazide groups orientated in close proximity to $\mathrm{K} 84$, with hydrogen bonds predicted to form between the ketone substituent of 4PEHz and K84 (Figure 4A). Enzyme preparations of PfPdx1 with $4 \mathrm{PEHz}$ revealed a concentration dependent decrease in PLP formation, with visible increases in a $320 \mathrm{~nm}$ chromophoric species (Figure 4B). Kinetic assays revealed $4 \mathrm{PEHz}$ as an effective inhibitor with an $\mathrm{IC}_{50}$ of $43 \pm 8 \mu \mathrm{M}$, whereas $\mathrm{EHz}$ was more than 20 times weaker (Figure $4 \mathrm{C}$, Table 2). Interestingly, when tested on the PfPdx1 component of the glutamine-dependent PLP synthase complex, containing PfPdx2, 4PEHz had an $\mathrm{IC}_{50}$ of $16 \pm 4 \mu \mathrm{M}$ (Figure 4D).

\section{Effect of $4 \mathrm{PEHz}$ and $\mathrm{EHz}$ on cellular level}

The effect of 4PEHz and EHz was determined on P. falciparum parasite proliferation. After $48 \mathrm{~h}$ incubation of intra-erythrocytic $P$. falciparum parasite cultures with $4 \mathrm{PEHz}$ an $\mathrm{IC}_{50}$ of $10.4 \pm 1.2 \mu \mathrm{M}$ was determined (Figure 5A). The unphosphorylated $\mathrm{EHz}$ was 13 -fold weaker compared to $4 \mathrm{PEHz}$, with an $\mathrm{IC}_{50}$ of $138 \pm 9 \mu \mathrm{M}$ (Figure 5A). To further elucidate the mode of action of $4 \mathrm{PEHz}$ in vivo the compound was tested on $P$. falciparum over-expressing $P f \mathrm{Pdx} 1 / P f \mathrm{Pdx} 2$ parasites [6]. Both mock control and PfPdx1/PfPdx 2 over-expressing parasite proliferation was monitored over a seven-day period in the presence of $1 \mu \mathrm{M} 4 \mathrm{PEHz}$. The proliferation of mock-parasites treated with $4 \mathrm{PEHz}$ significantly decreased over the course of $168 \mathrm{~h}$ (Figure 5B). Relative to control mock parasites the proliferation was significantly ( $\mathrm{p}$ $<0.05$ ) affected by $4 \mathrm{PEHz}$ only after $48 \mathrm{~h}$, and the parasite growth diminished to $10 \%$ of control parasites after $168 \mathrm{~h}$. In contrast, parasites overexpressing PfPdx1 and PfPdx2 were not significantly affected by $4 \mathrm{PEHz}$ treatment, and showed a relatively unchanged growth profile up until $144 \mathrm{~h}$. After $168 \mathrm{~h}$ the growth of the PfPdx 1/PfPdx 2 over-expressing parasite growth was not significantly different from untreated control parasites. This suggested that complementation of PLP biosynthesis through over-expression of PfPdx1 and PfPdx 2 protects the parasites from the effects of $4 \mathrm{PEHz}$.

Additionally, the growth rate constants $(\mathrm{K})$ of mock and PfPdx1/PfPdx2 over-expressing parasites were compared using logarithmic growth curves (Suppl. figure 2). The slopes of the fitted lines were used to calculate the growth rate constant which represents the increase in parasite growth per unit time. The growth rate constant $(\mathrm{K})$ of the mock parasites treated with $4 \mathrm{PEHz}$ was smaller than that of untreated-mock parasites. Statistical analyses comparing the 
slopes of the mock treated and mock-untreated fitted lines revealed a significant difference with accompanying analysis of covariance (ANCOVA) confirming this observation. This indicates that $4 \mathrm{PEHz}$ had an effect of parasite growth. In contrast, PfPdx1 and PfPdx2 overexpressing parasites had similar growth rate constants, and the slope of the fitted lines were not statistically different, indicating that the growth rate of the PfPdx1/PfPdx2-overexpressing parasites was unaffected by $4 \mathrm{PEHz}$-treatment. The effect of $4 \mathrm{PEHz}$ on the parasite life-stage composition was also investigated (Suppl. figure 3 and 4). During the seven day treatment period with $4 \mathrm{PEHz}$ the ring and trophozoite parasites from mock treated parasites had significantly different growth compositions on a single day when compared to untreated mock parasites (Suppl. figure 3). In contrast, the PfPdx1/PfPdx2 parasite life-stage compositions remained relative unchanged and no significant differences in parasite stage growth was observed (Suppl. figure 4). The complementation of PLP biosynthesis rescued the parasites from the effects of $4 \mathrm{PEHz}$, and also suggests that $4 \mathrm{PEHz}$ affects PfPdx1-related processes within the parasites.

\section{Discussion}

With antimalarial drugs being rendered ineffective at alarming rates newer chemical leads aimed at targeting diverse metabolic pathways are required. The malaria parasite depends on de novo biosynthesis of PLP by PLP synthase and the cofactor plays an essential role during amino acid metabolism and folate biosynthesis [7]. PLP-dependent enzymes involved in amino acid biosynthesis principally bind PLP via an imine bond or Schiff base through utilizing an active site lysine residue [33]. PLP has recently been ascribed potent antioxidant roles and has been proposed to aid in reducing parasite oxidative stress associated with heme and free-iron release during digestion of host erythrocyte hemoglobin [6, 34]. The components of the PLP synthase are up-regulated when parasites are exposed to singlet molecular oxygen $\left({ }^{1} \mathrm{O}_{2}\right)$, and through protein complementation PfPdx1 and PfPdx2 were directly associated with detoxification of ${ }^{1} \mathrm{O}_{2}$ [6]. PLP biosynthesis is indispensable to the parasites and this pathway is also absent from humans making it an ideal target for the development of chemotherapeutics.

Three distinct sites for binding of the reaction substrates and products have been identified on Pdx1. Crystallographic evidence for the R5P binding site was provided from Thermotoga maritima Pdx1 (TmPdx1) with Ru5P bound via an imine to the K82 residue (equivalent to PfPdx1 K83) [31]. In the Saccharomyces cerevisiae Pdx1 structure an additional site, previously observed to contain a phosphate ion, was shown to accommodate PLP $[12,13,31]$. Residues of the PLP binding site in PfPdx 1 include the highly conserved ERR triad comprising E136, R139 and R140, which interact mainly with the phosphate groups of PLP $[13,24]$. Moreover, a site which contained G3P was identified and involved R164 (equivalent to R167 in PfPdx1) [13]. The R5P, PLP and G3P binding sites are located more than $10 \AA$ from each other and, even though much is known regarding the reaction mechanism, some questions regarding substrate mobility and dynamics from these distant binding sites remains to be uncovered.

The Pdx1 protein of PLP synthase is highly conserved, and could imply evolutionary maintenance of specialized function [34, 35]. Mechanistic elucidation of the Pdx1 reaction coordinate has highlighted the multitude of complex mechanistic steps involved in forming PLP $[10,11,15]$. The reaction mechanism consists of several imine bond formations [10]. Initial binding of R5P entails formation of an imine with a Pdx1 lysine residue (Figure 6) followed by isomerization into a Ru5P imine adduct. The bound intermediate is subsequently converted into the $\mathrm{I}_{320}$ chromophore, which forms an imine bond with G3P, and undergoes ring closure to ultimately form PLP (Figure 6) [10]. Both substrates R5P (acyclic-forms) and 
G3P have reactive $\mathrm{C} 1$ aldehyde groups and are phosphorylated. The $\alpha$-hydroxy or $\mathrm{C} 1$ aldehyde group on both R5P and G3P is essential for initial substrate binding, $\mathrm{I}_{320}$ formation as well as G3P-binding. The G3P-phosphate group forms part of the final PLP molecule, whereas the R5P-phosphate group is eliminated during the reaction mechanism (Figure 6) [10]. In TmPdx 1 the R5P-phosphate group was shown to form $\mathrm{H}$-bonds with three glycine amide nitrogen atoms and could function to facilitate orientation of the molecule during catalytic conversion [13].

We sought to identify molecules which could be accommodated within the R5P active site of PfPdx1, possibly affecting PLP formation, which would make attractive starting points for the development of novel antimalarials. Virtual screening of the PfPdx1 R5P-binding site pharmacophores identified compounds with diverse chemical scaffolds. Several polyhydroxylated compounds with similar sugar-like backbone to R5P were identified, however were not effective at inhibiting PfPdx1. Compounds with terminal sulfonate groups, which were predicted to mimic phosphate groups of R5P in the R5P-binding cavity were ineffective at inhibiting PfPdx1. The reasons for this became clear when we tested closelyrelated analogues of R5P, which were more effective at inhibiting PfPdx1. Initial binding to PfPdx1 requires Schiff-base adduct or imine formation with internal K-residues, and computationally-predicted compounds were not capable of this due to the lack of reactive functional groups.

DR5P inhibited PfPdx1, and marginally reduced formation of $\mathrm{I}_{320}$ suggesting that DR5P may compete with R5P, albeit weakly. Following the currently proposed reaction mechanism of PfPdx1, DR5P would be accommodated by PfPdxland potentially bind to the K83 residue [10]. The DR5P-adduct might not undergo conversion due to the lack of the C2 hydroxyl substituent, which could affect the resident time within the active site, possibly explaining the lack of potency of DR5P. E4P effectively inhibited PfPdx1. Structurally E4P is similar to R5P, with one less carbon and hydroxyl substituent. We speculated that E4P could be interfering with R5P binding in PfPdx1. Docking simulations predicted that E4P could occupy the R5P-binding site and that the sugar-derived hydroxyl substituents as well as phosphate moiety have favourable H-bonding interactions within this site. E4P could outcompete R5P for K83 imine formation, and potentially also undergo conversion similar to R5P (Figure 6). This would affect binding of R5P and lead to disrupted PLP formation. The E4P-analogue DXP, with a terminal ketone group, was not an effective inhibitor. This suggested the $\mathrm{C} 1$ aldehyde reactivity, as found on E4P, was important for inhibition and this feature could possibly determine entry into the R5P active site. D-erythrose was almost 50times weaker than E4P suggesting that the phosphate group is also essential for efficacy. Similar to R5P, the phosphate group of E4P might facilitate orientation of the molecule in the R5P-binding site. The structure-activity relationship of E4P and analogues suggested that both the terminal $\alpha$-hydroxy $\mathrm{C} 1$ aldehyde- and hydrolyzable phosphate-group arrangements were important for effective inhibition of PfPdx1.

Spinach RPI is inhibited by E4P, and more so by 4-phosphoerythronic acid [36]. The weak RPI activity of PfPdx1 could therefore similarly be targeted by such molecules. Glucose-6phosphate isomerases (GPI) are inhibited by acyclic phospho-sugars which have free carbonyl groups, like E4P [37-39]. Moreover, the GPI active site resembles that of triosephosphate isomerases (TIM) - a function that has been implicated in Pdx1 proteins [40, 41]. Even though no definitive site for DHAP isomerization has been found, the implicated TIMfunction of PfPdx1, albeit weak, could be affected by E4P [13, 14]. Therefore an alternative mode of action of E4P could involve binding to the postulated G3P-binding site [13]. E4P might interact with K120 (equivalent to $S c \mathrm{Pdx} 1 \mathrm{~K} 117$ ), whereby E4P would mimic G3P, potentially interfering with downstream PLP ring-closure steps. E4P could therefore either affect the RPI- or TIM-activities in PfPdx1. 
Our studies suggest for the first time that the naturally occurring E4P metabolite could regulate PLP production in $P$. falciparum parasites through inhibition of PfPdx1. Considering that the DXP-dependent pathway in E. coli utilizes the E4P precursor for the production of PLP [42], it is interesting from an evolutionary point of view that this molecule inhibits the plasmodial Pdx1 in the DXP-independent pathway. E4P is a vital precursor for the production of chorismate, and downstream phenylalanine, tyrosine and $p$-aminobenzoic acid, utilized for the production of folates [43]. The pathway for the production of E4P from sedoheptulose 7phosphate by transaldolases has not been identified in $P$. falciparum [44], however transketolases are known to produce E4P from F6P and G3P [45]. E4P is an indispensable metabolite to the parasites and future studies might aim to explore the in vivo effects of $\mathrm{E} 4 \mathrm{P}$ fluctuations on PLP production.

From the $T m \mathrm{Pdx} 1$ structure it was shown that $\mathrm{D} 26$ and $\mathrm{K} 83$ constitute the R5P binding site, therefore could be directly involved in $\mathrm{I}_{320}$ formation $[31,46]$. Our observations for the DKK PfPdx 1 mutant confirmed previous results, and residues involved in $\mathrm{I}_{320}$ formation are most probably D26 and K83 in PfPdx1 [15, 31]. Moreover, our findings support previous results which showed that the $\Delta \mathrm{C}$-terminal Pdx1 mutant is incapable of forming $\mathrm{I}_{320}$ [30]. Residues R139 and R140 of the ERR motif forms part of the PLP binding site [13, 31, 46]. Mutation of these residues was previously shown to abolish PfPdx1 activity, underscoring their catalytic importance most likely during PLP binding and release [24]. Our observations revealed that the ERR PfPdx1 variant was still able to form $\mathrm{I}_{320}$. This suggested that these residues were not directly involved in R5P isomerization leading to formation of the chromophore. The highly conserved residues R85, H88 and E91 from the RHE motif of PfPdx1, located on the loop between $\beta 3$ and $\alpha 3$, are essential for Pdx 1 hexamer assembly and subsequent $\operatorname{Pdx} 2$ binding and activation [24]. These residues were suggested to facilitate Pdx1 hexameric pre-assembly, which is coordinated and a prerequisite for PLP synthase activation [24]. In the absence of PfPdx2, the RHE triple mutant was also incapable of forming the $\mathrm{I}_{320}$ specie. Lack of $\mathrm{I}_{320}$ formation in the RHE mutant indicates that these residues are intricately involved in R5P binding, additionally taking part in active site reorganisation during dodecamer assembly. A glycine residue (G84) located on the same loop region as these RHE residues was shown to form H-bonds with D110 and a water molecule which forms part of the G3P binding site [13]. This suggests that the RHE-containing loop region is vital for both assembly and enzyme activity and emphasizes that Pdx1:Pdxl interactions, involving these RHE residues, could serve as a target site for the development of novel target-based inhibitors.

The moderate inhibitory efficacy of E4P against PfPdx1 motivated testing of E4P analogues against the enzyme. 4PEHz inhibited PfPdx 1 and the entire PLP synthase complex with low micromolar efficacy. During oligomerization of $\mathrm{Pdx} 1$ and $\mathrm{Pdx} 2$ into the functional PLP synthase Pdx1 activates the glutaminase activity in Pdx2 monomers leading to the formation of a putative opening through which ammonia is channelled [12]. Oligomerization also leads to increased R5P binding affinity in Pdx1 [47]. We noted increased inhibitory efficacy of 4PEHz in the PfPdx1:PfPdx2 complex suggesting improved binding interactions of 4PEHz, similar to previous observations for R5P, and thus that 4PEHz may bind in the R5P binding site. 4PEHz was previously reported as a weak inhibitor of spinach RPI with a $K_{i}$ of $1.8 \pm 0.2$ $\mathrm{mM}$ [32]. 4PEHz structurally mimics the 1,2-cis-enediolate intermediate in the RPI isomerization step [32]. The lead us to speculate that 4PEHz could affect the RPI activity of PfPdx1. Both EHz and 4PEHz can potentially form Schiff-bases with PLP through linkage with hydrazide groups. However, the difference in inhibitory efficiency, indicated by involvement of the phosphate group, suggests that the enzyme preferentially allows entry of more negatively charged molecules like 4PEHz. Similar observations for E4P compared to Derythrose also support this hypothesis. In both E4P and 4PEHz the presence of hydrolyzable phosphate groups improved inhibitory activity and suggests that these two molecules could similarly occupy the R5P binding site on the protein, which is also supported by in silico 
docking results. As outlined in Figure 6, 4PEHz shares chemical similarities to both E4P and $\mathrm{R} 5 \mathrm{P}$, suggesting a similar mode of action compared to E4P.

$4 \mathrm{PEHz}$ inhibited parasite growth at low micromolar concentrations, compared to the unphosphorylated $\mathrm{EHz}$ analogue, which was at least 12-fold weaker. PLP is an essential cofactor, and continuously recycled within the parasite, therefore expectedly attenuation of PLP biosynthesis has a dramatic effect on parasite proliferation. The complementation of $P f \mathrm{Pdx} 1$ and PfPdx2 in P. falciparum parasites in vivo was shown to increase tolerance to cercosporin-induced oxidative stress $[6,48]$. Here we similarly observed that parasites which over-express $P f \mathrm{Pdx} 1$ and $P f \mathrm{Pdx} 2$ were not affected by the PfPdx1-inhibtor 4PEHz. In contrast, parasites harbouring the same expression plasmid, without $P f \mathrm{Pdx} 1$ and $P f \mathrm{Pdx} 2$, had reduced growth rates and were significantly attenuated by $4 \mathrm{PEHz}$. Almost 3-fold greater PLP levels were previously reported in PfPdx1/PfPdx2 complemented parasites [6]. This complementation of PLP synthesis in vivo counteracts the effects of $4 \mathrm{PEHz}$, and suggests that $4 \mathrm{PEHz}$ interferes primarily with this part of the parasite metabolism. These results support $4 \mathrm{PEHz}$ as a novel lead compound for targeting vitamin $\mathrm{B}_{6}$ biosynthesis within the malaria parasite.

Endogenous pyridoxal/pyridoxine salvage pathways might be capable of complementing the PLP pool in the parasites and could diminish the effects of PfPdxl inhibitors. Indeed the parasite possesses a functional pyridoxal kinase $(\mathrm{PdxK})$, which activates salvaged $\mathrm{B}_{6}$ vitamers. This pathway has successfully been exploited using prodrugs which are trapped in the parasite upon phosphorylation by PdxK [29]. Once phosphorylated these PLP analogues were shown to disrupt PLP-dependent processes and thus kill the parasite selectively [29]. However, pyridoxal is bound to hemoglobin and such not available for uptake. Furthermore the parasite shows no growth defect when cultured in pyridoxine free medium [7]. Since $4 \mathrm{PEHz}$ inhibits parasite proliferation in culture media containing approximately $5 \mu \mathrm{M}$ pyridoxine this suggests that $4 \mathrm{PEHz}$ is effective irrespective of endogenous $\mathrm{B}_{6}$ salvage pathways. Nevertheless, in combination with this strategy, compounds that directly target PfPdx1 may contribute even more effectively by additionally starving the parasites of PLP and thus leading to parasite death.

Novel lead scaffolds were identified against PfPdx1, and were capable of targeting PLP biosynthesis within the $P$. falciparum parasites. Lead compounds should ideally have greater potency, and undoubtedly these compounds will have to undergo further optimization to improve their efficacy. These relatively simplistic, low-molecular weight compounds are well suited for additional molecular weight contributions from chemical modifications or functionalization during scaffold modifications. These compounds had structural complementarity to $\mathrm{R} 5 \mathrm{P}$ and more noticeably contained relatively reactive $\mathrm{C} 1$ groups. Compounds containing reactive functional groups are undesirable optimization starting points and would normally have been removed from high-throughput or virtual screening libraries $[49,50]$. A successful antimalarial with a similar chemical structure is the isoprenoid precursor pathway inhibitor fosmidomycin, with terminal aminoaldehyde arrangement [51, 52]. The nature of PfPdx1, its substrates, and the reaction mechanism also needs to be considered in order to achieve better inhibitor efficacy. Additionally the mode and site of binding in PfPdx1 will need to be determined, and may provide insights into the mode of action of these inhibitors.

\section{Acknowledgments}

S. R. conducted part of this work in fulfilment of the requirements for a Ph.D. from the University of Pretoria.

\section{Author contributions}


Devised experimental procedures and strategies: SR IM PB EB LS AL LB CW. Conducted experiments: SR IM CW. Manuscript preparation: IM PB EB LS AL LB CW.

\section{Funding}

This work was funded by the grants [WR 124/2] and [WR 124/3] from the Deutsche Forschungsgemeinschaft (DFG) to CW as well as the National Research Foundation (NRF) of South Africa [65876] and NRF-DFG Scientific exchange grants to LMB, IM and CW. SBR was supported by the $\mathrm{PhD}$ sandwich programme of the German Academic Exchange Programme (DAAD) [A/08/99008]. CW is supported by grant [2009/54325-2] from the Fundação de Amparo à Pesquisa do Estado de São Paulo (FAPESP) within the "Jovem Pesquisador" programme.

\section{References}

1 Greenwood, B. M., Fidock, D. A., Kyle, D. E., Kappe, S. H. I., Alonso, P. L., Collins, F. H. and Duffy, P. E. (2008) Malaria: progress, perils, and prospects for eradication. J. Clin. Invest. 118, 1266-1276

2 Greenwood, B. M. (2008) Control to elimination: implications for malaria research. Trends Parasitol. 24, 449-454

3 Guiguemde, W. A., Shelat, A. A., Garcia-Bustos, J. F., Diagana, T. T., Gamo, F. J. and Guy, R. K. (2012) Global Phenotypic Screening for Antimalarials. Chem. Biol. 19, 116-129

4 Eliot, A. C. and Kirsch, J. F. (2004) Pyridoxal phosphate enzymes: mechanistic, structural, and evolutionary considerations. Annu. Rev. Biochem. 73, 383-415

5 Wrenger, C., Eschbach, M., Muller, I. B., Warnecke, D. and Walter, R. D. (2005) Analysis of the vitamin B6 biosynthesis pathway in the human malaria parasite Plasmodium falciparum. J. Biol. Chem. 280, 5242-5248

6 Knöckel, J., Müller, I. B., Butzloff, S., Bergmann , B., Walter, R. D. and Wrenger, C. (2012) The antioxidative effect of de novo generated vitamin B6 in Plasmodium falciparum validated by protein interference. Biochem. J. 443, 397-405

7 Müller, I. B., Hyde, J. E. and Wrenger, C. (2010) Vitamin B metabolism in Plasmodium falciparum as a source of drug targets. Trends Parasitol. 26, 35-43

8 Cane, D. E., Du, S., Robinson, J. K., Hsiung, Y. and Spenser, I. D. (1999) Biosynthesis of vitamin B6: enzymatic conversion of 1-deoxy-D-xylulose-5-phosphate to pyridoxol phosphate. J. Am. Chem. Soc. 121, 7722-7723

9 Knöckel, J., Müller, I. B., Bergmann, B., Walter, R. D. and Wrenger, C. (2007) The apicomplexan parasite Toxoplasma gondii generates pyridoxal phosphate de novo. Mol. Biochem. Parasitol. 152, 108-111

10 Hanes, J. W., Keresztes, I. and Begley, T. P. (2008) 13C NMR snapshots of the complex reaction coordinate of pyridoxal phosphate synthase. Nat. Chem. Biol. 4, 425-430

11 Gengenbacher, M., Fitzpatrick, T. B., Raschle, T., Flicker, K., Sinning, I., Müller, S., Macheroux, P., Tews, I. and Kappes, B. (2006) Vitamin B6 biosynthesis by the malaria parasite Plasmodium falciparum. J. Biol. Chem. 281, 3633-3641

12 Strohmeier, M., Raschle, T., Mazurkiewicz, J., Rippe, K., Sinning, I., Fitzpatrick, T. B. and Tews, I. (2006) Structure of a bacterial pyridoxal 5'-phosphate synthase complex. Proc. Natl. Acad. Sci. USA. 103, 19284-19289 
13 Zhang, X., Teng, Y., Liu, J., He, Y., Zhou, K., Chen, Y. and Zhou, C. (2010) Structural insights into the catalytic mechanism of the yeast pyridoxal 5-phosphate synthase Snz1. Biochem. J. 432, 445-450

14 Hanes, J. W., Burns, K. E., Hilmey, D. G., Chatterjee, A., Dorrestein, P. C. and Begley, T. P. (2008) Mechanistic studies on pyridoxal phosphate synthase: the reaction pathway leading to a chromophoric intermediate. J. Am. Chem. Soc. 130, 3043-3052

15 Raschle, T., Arigoni, D., Brunisholz, R., Rechsteiner, H., Amrhein, N. and Fitzpatrick, T. B. (2007) Reaction mechanism of pyridoxal 5'-phosphate synthase. Detection of an enzyme-bound chromophoric intermediate. J. Biol. Chem. 282, 6098-6105

16 Guédez, G., Hipp, K., Windeisen, V., Derrer, B., Gengenbacher, M., Böttcher, B., Sinning, I., Kappes, B. and Tews, I. (2012) Assembly of the eukaryotic PLP-synthase complex from Plasmodium and activation of the Pdx1 enzyme. Structure. 20, 172-184

17 Laskowski, R. A. (2001) PDBsum: summaries and analyses of PDB structures. Nucleic Acids Res. 29, 221-222

18 Laskowski, R. A. (2009) PDBsum new things. Nucleic Acids Res. 37, D355-D359

19 Irwin, J. J. and Shoichet, B. K. (2005) ZINC-a free database of commercially available compounds for virtual screening. J. Chem. Inf. Model. 45, 177-182

20 Venkatachalam, C., Jiang, X., Oldfield, T. and Waldman, M. (2003) LigandFit: a novel method for the shape-directed rapid docking of ligands to protein active sites. $\mathrm{J}$. Mol. Graphics Model. 21, 289-307

21 Mayo, S. L., Olafson, B. D. and Goddard, W. A. (1990) DREIDING: A generic force field for molecular simulations. J. Phys. Chem. 94, 8897-8909

22 Krammer, A., Kirchhoff, P. D., Jiang, X., Venkatachalam, C. and Waldman, M. (2005) LigScore: a novel scoring function for predicting binding affinities. J. Mol. Graphics Model. 23, 395-407

23 Humphrey, W., Dalke, A. and Schulten, K. (1996) VMD: visual molecular dynamics. J. Mol. Graphics. 14, 33-38

24 Müller, I. B., Knöckel, J., Groves, M. R., Jordanova, R., Ealick, S. E., Walter, R. D. and Wrenger, C. (2008) The assembly of the plasmodial PLP synthase complex follows a defined course. PLoS ONE. 3, 1-9

25 Knöckel, J., Jordanova, R., Müller, I. B., Wrenger, C. and Groves, M. R. (2009) Mobility of the conserved glycine 155 is required for formation of the active plasmodial dodecamer. Biochim. Biophys. Acta. 1790, 347-350

26 Trager, W. and Jensen, J. B. (1976) Human malaria parasites in continuous culture. Science. 193, 673-675

27 Das Gupta, R., Krause-Ihle, T., Bergmann, B., Müller, I. B., Khomutov, A. R., Müller, S., Walter, R. D. and Lüersen, K. (2005) 3-Aminooxy-1-aminopropane and derivatives have an antiproliferative effect on cultured Plasmodium falciparum by decreasing intracellular polyamine concentrations. Antimicrob. Agents Chemother. 49, 28572864

28 Lambros, C. and Vanderberg, J. P. (1979) Synchronization of Plasmodium falciparum erythrocytic stages in culture. J. Parasitol. 3, 418-420

29 Müller, I. B., Wu, F., Bergmann , B., Knöckel, J., Gehring, H., Walter, R. D. and Wrenger, C. (2009) Poisoning pyridoxal 5-phosphate dependent enzymes: a new strategy to target the malaria parasite Plasmodium falciparum. PLoS ONE. 4, 1-9

30 Derrer, B., Windeisen, V., Guedez Rodriguez, G., Seidler, J., Gengenbacher, M., Lehmann, W. D., Rippe, K., Sinning, I., Tews, I. and Kappes, B. (2010) Defining the structural requirements of ribose 5-phosphate-binding and intersubunt cross-talk of the malarial pyridoxal 5-phopshate synthase. FEBS Lett. 584, 4169-4174 
31 Zein, F., Zhang, Y., Kang, Y. N., Burns, K., Begley, T. P. and Ealick, S. E. (2006) Structural insights into the mechanism of the PLP synthase holoenzyme from Thermotoga maritima. Biochemistry. 45, 14609-14620

32 Burgos, E. and Salmon, L. (2004) Synthesis and evaluation of new 4-phospho-Derythronic acid derivatives as competitive inhibitors of spinach ribose-5-phosphate isomerase. Tetrahedron Lett. 45, 753-756

33 Christen, P. and Mehta, P. K. (2001) From cofactor to enzymes. The molecular evolution of pyridoxal-5'-phosphate-dependent enzymes. Chem. Rec. 1, 436-447

34 Ehrenshaft, M., Bilski, P., Li, M. Y., Chignell, C. F. and Daub, M. E. (1999) A highly conserved sequence is a novel gene involved in de novo vitamin B6 biosynthesis. Proc. Natl. Acad. Sci. USA. 96, 9374-9378

35 Galperin, M. Y. and Koonin, E. V. (1997) Seqeunce analysis of an exceptionally conserved operon suggests enzymes for a new link beteen histidine and purine biosynthesis. Mol. Microbiol. 24, 443-445

36 Woodruff, W. and Wolfenden, R. (1979) Inhibition of ribose-5-phosphate isomerase by 4-phosphoerythronate. J. Biol. Chem. 254, 5866

37 Backhausen, J. E., Jöstingmeyer, P. and Scheibe, R. (1997) Competitive inhibition of spinach leaf phosphoglucose isomerase isoenzymes by erythrose 4-phosphate. Plant Sci. 130, 121-131

38 Chirgwin, J., Parsons, T. and Noltmann, E. (1975) Mechanistic implications of the pH independence of inhibition of phosphoglucose isomerase by neutral sugar phosphates. J. Biol. Chem. 250, 7277

39 Ruijter, G. J. G. and Visser, J. (1999) Characterization of Aspergillus niger phosphoglucose isomerase. Use for quantitative determination of erythrose 4phosphate. Biochimie. 81, 267-272

40 Jeffery, C. J., Bahnson, B. J., Chien, W., Ringe, D. and Petsko, G. A. (2000) Crystal structure of rabbit phosphoglucose isomerase, a glycolytic enzyme that moonlights as neuroleukin, autocrine motility factor, and differentiation mediator. Biochemistry. 39, 955-964

41 Burns, K. E., Xiang, Y., Kinsland, C. L., McLafferty, F. W. and Begley, T. P. (2005) Reconstitution and biochemical characterization of a new pyridoxal-5'-phosphate biosynthetic pathway. J. Am. Chem. Soc. 127, 3682-3683

42 Fitzpatrick, T. B., Amrhein, N., Kappes, B., Macheroux, P., Tews, I. and Raschle, T. (2007) Two independent routes of de novo vitamin $\mathrm{B}_{6}$ biosynthesis: not that different after all. Biochem. J. 407, 1-13

43 Roberts, C. W., Roberts, F., Lyons, R. E., Kirisits, M. J., Mui, E. J., Finnerty, J., Johnson, J. J., Ferguson, D. J. P., Coggins, J. R., Krell, T., Coombs, G. H., Milhous, W. K., Kyle, D. E., Tzipori, S., Barnwell, J., Dame, J. B., Jane, C. and McLeod, R. (2002) The Shikimate Pathway and Its Branches in Apicomplexan Parasites. J. Infect. Dis. 185, S25-S36

44 Bozdech, Z. and Ginsburg, H. (2005) Data mining of the transcriptome of Plasmodium falciparum: the pentose phosphate pathway and ancillary processes. Malaria J. 4, 17

45 Joshi, S., Singh, A. R., Kumar, A., Misra, P. C., Siddiqi, M. I. and Saxena, J. K. (2008) Molecular cloning and characterization of Plasmodium falciparum transketolase. Mol. Biochem. Parasitol. 160, 32-41

46 Moccand, C., Kaufmann, M. and Fitzpatrick, T. B. (2011) It takes two to tango: defining an essential second active site in pyridoxal 5'-phosphate synthase. PLoS ONE. 6, e16042

47 Raschle, T., Speziga, D., Kress, W., Moccand, C., Gehrig, P., Amrhein, N., WeberBan, E. and Fitzpatrick, T. B. (2009) Intersubunit cross-talk in pyridoxal 5'-phosphate synthase coordinated by the $\mathrm{C}$ terminus of the synthase subunit. J. Biol. Chem. 284, 7706-7718 
48 Butzloff, S., Groves, M. R., Wrenger, C. and Müller, I. B. (2012) Cytometric quantification of singlet oxygen in the human malaria parasite Plasmodium falciparum. Cytometry A. 81, 698-703

49 Davis, A. M., Keeling, D. J., Steele, J., Tomkinson, N. P. and Tinker, A. C. Components of Successful Lead Generation. Curr. Top. Med. Chem. 5, 421-439

50 Zhao, H. (2007) Scaffold selection and scaffold hopping in lead generation: a medicinal chemistry perspective. Drug Discov. Today. 12, 149-155

51 Oyakhirome, S., Issifou, S., Pongratz, P., Barondi, F., Ramharter, M., Kun, J. F., Missinou, M. A., Lell, B. and Kremsner, P. G. (2007) Randomized controlled trial of fosmidomycin-clindamycin versus sulfadoxine-pyrimethamine in the treatment of Plasmodium falciparum malaria. Antimicrob. Agents Chemother. 51, 1869-1871

52 Borrmann, S., Issifou, S., Esser, G., Adegnika, A. A., Ramharter, M., Matsiegui, P.-B., Oyakhirome, S., Mboumba, D. P. M., Missinou, M. A., Kun, J. F. J., Jomaa, H. and Kremsner, P. G. (2004) Fosmidomycin-clindamycin for the treatment of Plasmodium falciparum malaria. J. Infect. Dis. 190, 1534-1540

\section{Tables and Figures}

Table 1: In silico-identified compounds tested against PfPdx1. Several pharmacophores generated for PfPdx1 were screened to obtain best-fitting ligands which were, together with rationally selected compounds, subjected to additional docking into PfPdx1 homology models. R5P was used as a control during docking simulations. Resultant compounds were tested on purified PfPdx1 (see the Experimental section). Values in parentheses indicate scores calculated for ligands docked into $P b P d x 1$. Values are from three or more independent experiments performed in duplicate, with the standard error of the mean (SEM) indicated.

\begin{tabular}{|c|c|c|c|c|c|c|}
\hline Compound & Structure & $\begin{array}{c}\text { LigScore } \\
1^{\mathrm{a}}\end{array}$ & $\begin{array}{c}\text { LigScore } \\
\mathbf{2}^{\mathrm{a}}\end{array}$ & $\begin{array}{l}\text { Dock } \\
\text { score }^{b}\end{array}$ & $\begin{array}{l}\text { Conc. } \\
\text { (mM) }\end{array}$ & $\begin{array}{c}\text { PfPdx1 } \\
\text { inhibition (\% } \\
\text { residual } \\
\text { activity) }\end{array}$ \\
\hline 1 & & 6.17 & 5.6 & 44.91 & 0.5 & $96 \pm 3$ \\
\hline 2 & & 5.75 & 5.39 & 61.35 & 0.5 & $103 \pm 4$ \\
\hline 3 & & 5.97 & 5.51 & 54.76 & 3 & $96 \pm 5$ \\
\hline 4 & & 5.22 & 4.94 & 82.18 & 3 & $96 \pm 3$ \\
\hline 5 & & 4.76 & 5.36 & 24.01 & 0.5 & $99 \pm 5$ \\
\hline
\end{tabular}




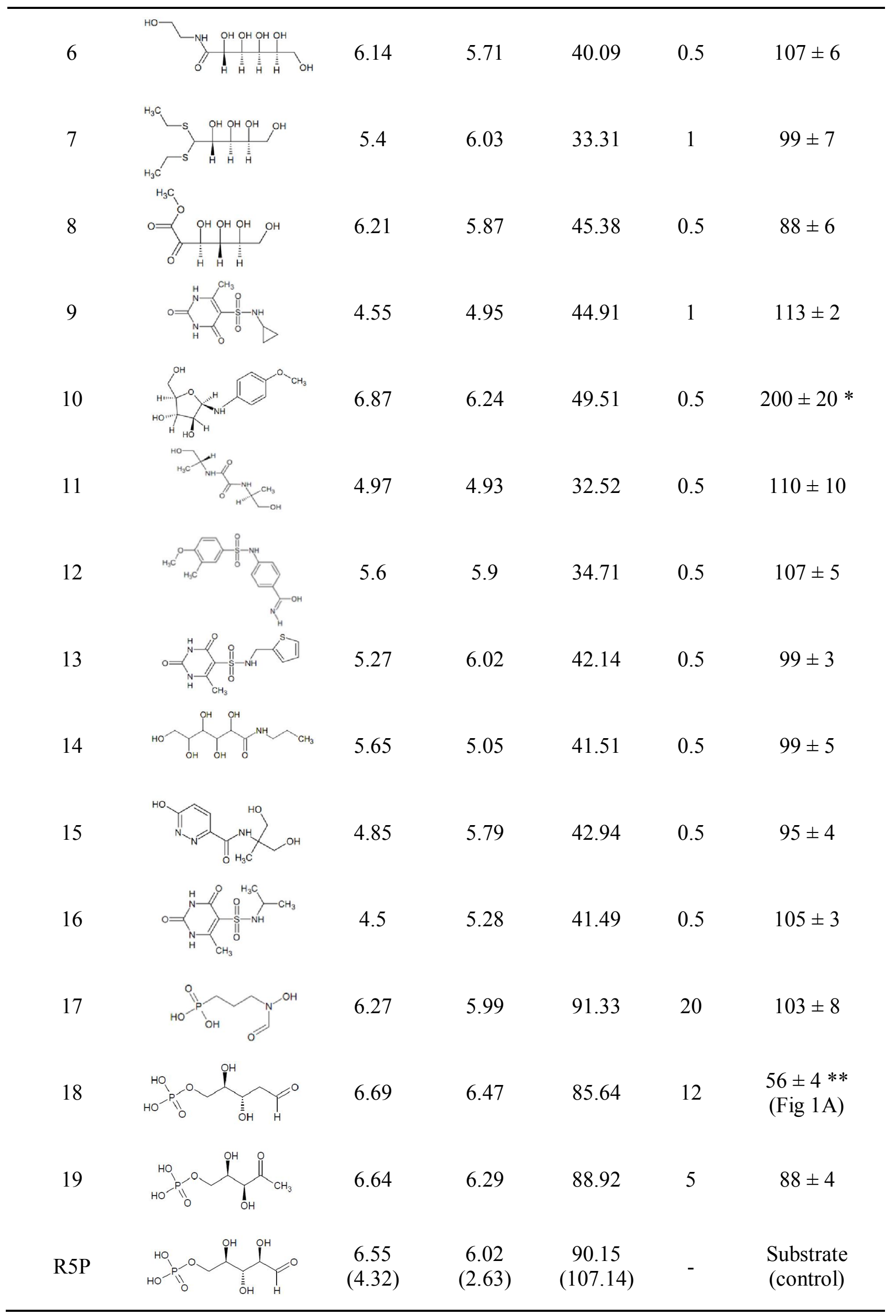




\begin{tabular}{lllllll}
\hline G3P & 5.87 & 5.36 & 82.83 & - & Substrate \\
PLP & & & & \\
Product
\end{tabular}

Footnotes: ${ }^{\mathrm{a}}$ LigScore1 and LigScore2, with units of $\mathrm{pKi}(-\log \mathrm{Ki})$, refers to predicted receptorligand binding affinities. ${ }^{b}$ Dockscore refers to the unitless rigid body minimization energy of the final ligand pose calculated during Monte Carlo trials. ${ }^{*}$ absorbance of compound interfered with detection of PLP. ${ }^{* *} P<0.10$ in a two-sided t-test.

Table 2: E4P analogues and their efficacy on PfPdx1. Selected compounds were docked into a PfPdx1 homology model and poses were evaluated using LigScore ligand pose scoring functions. R5P was used as a control during docking simulations, with Ligscore and Dockscore values listed in Table 1. Values in parentheses indicate scores calculated for ligands in $P b P d x 1 . \mathrm{IC}_{50}$ values were calculated from dose-response curves of the respective compounds incubated with purified PfPdx1, as reported in the Experimental section. $\mathrm{IC}_{50}$ values represent data from three of more independent experiments performed in duplicate, with the SEM indicated.

\begin{tabular}{|c|c|c|c|c|c|}
\hline Compound & Structure & $\underset{a}{\operatorname{LigScore} 1}$ & $\underset{a}{\operatorname{LigScore} 2}$ & $\begin{array}{c}\text { Dock } \\
\text { score }^{b}\end{array}$ & $\mathrm{IC}_{50}$ on $P f \mathrm{Pdx} 1$ \\
\hline E4P & & $6.40(3.00)$ & $6.09(0.41)$ & $\begin{array}{c}85.17 \\
(120.89)\end{array}$ & $3.7 \pm 0.9 \mathrm{mM}$ \\
\hline D-erythrose & & 4.74 & 4.25 & 42.19 & $160 \pm 46 \mathrm{mM}$ \\
\hline $\mathrm{EHz}$ & & 5.34 & 5.39 & 53.48 & $902 \pm 206 \mu \mathrm{M}$ \\
\hline 4PEHz & & $6.55(3.73)$ & $6.43(1.46)$ & $\begin{array}{c}98.96 \\
(117.01)\end{array}$ & $43 \pm 8 \mu \mathrm{M}$ \\
\hline
\end{tabular}

Footnotes: ${ }^{a}$ LigScore1 and LigScore2, with units of pKi (-log Ki), refers to predicted receptorligand binding affinities. ${ }^{b}$ Dockscore refers to the unitless rigid body minimization energy of the final ligand pose calculated during Monte Carlo trials. 
Figure 1. Effect of alternative sugar substrates on PfPdx1. (A) Inhibition of PfPdx1 by DR5P. Representative UV-visible absorbance spectra of PfPdx1 incubated with increasing concentrations of DR5P. Decreased levels of PLP, visible as diminished $414 \mathrm{~nm}$ PLP-Schiff base absorbance, correlated with increased concentrations of DR5P. (B) potential utilisation of alternative sugars by PfPdx1. Representative UV-visible absorbance spectra of PfPdx1 incubated with $3 \mathrm{mM}$ R5P or E4P, and $12 \mathrm{mM}$ F6P or DR5P in the presence of G3P. Only R5P supported formation of PLP visible at $414 \mathrm{~nm}$, and in the absence of G3P (- G3P) the I 320 specie could be formed from R5P. A $320 \mathrm{~nm}$ maximum absorbance peak was observed for E4P. (C) R5P docked into PbPdx1 using LigandFit. The ligand poses or receptor-ligand binding affinities were calculated using LigScore1, with units of $\mathrm{pK}_{\mathrm{i}}\left(-\log \mathrm{K}_{\mathrm{i}}\right)$, in which R5P could be docked back into the structure with a score of 4.32. (D) E4P docked into the R5P binding site of $P b P d x 1$. The molecule was predicted to hydrogen bond with K84, mainly involving the aldehyde and hydroxyl groups, similary interacting with residue D27. E4P was calculated to have a LigScore1 of 3.00.

Figure 2. Inhibitory effect of E4P on PfPdx1. (A) Representative UV-visible absorbance spectra of PfPdx1 incubated with increasing concentrations of E4P, which affected both PLP production and the formation of $\mathrm{I}_{320}$. (B) $\mathrm{IC}_{50}$ of E4P and D-erythrose. The $\mathrm{IC}_{50}$ of E4P was calculated as $3.7 \pm 0.9 \mathrm{mM}$ and D-erythrose as $160 \pm 46 \mathrm{mM}$. Values represent data from three independent experiments performed in duplicate compared to an uninhibited control, error bars represent the standard error of the mean (SEM) given with the $95 \%$ confidence interval in dotted lines.

Figure 3. Formation of R5P-related $I_{320}$ with PfPdx1 variants. (A) Wild-type (WT), DKK (D26A, K83A and K151A) and ERR (E136A, R139A and R140A) variants of PfPdx1 were incubated with $0.5 \mathrm{mM} \mathrm{R} 5 \mathrm{P}$ and $20 \mathrm{mM} \mathrm{NH}_{4} \mathrm{Cl}$. $100 \mu \mathrm{g}$ of each $\mathrm{Pdx} 1$ variant was present, except for the DKK mutant represented with $19.8 \mu \mathrm{g}$ protein. Positive control reactions WT (R5P and G3P) included $0.5 \mathrm{mM} \mathrm{G3P}$ to demonstrate active protein, with PLP formation visible at $414 \mathrm{~nm}$. $\mathrm{I}_{320}$ formation was evident in WT (R5P) protein which was solely incubated with R5P. The ERR PfPdx1 (R5P) mutant showed formation of $\mathrm{I}_{320}$, whereas the DKK mutant did not form this species underscoring the importance of the DKK residues. (B) WT, $\Delta \mathrm{C}$ and RHE (R85A, H88A and E91A) PfPdx1 variants were incubated with R5P. $100 \mu \mathrm{g}$ of each protein variant was used in the assay. In both $\triangle \mathrm{C}$ and RHE $\mathrm{I}_{320}$ formation was abolished. (C) Activity of WT and R167A PfPdx1. The assay contained $100 \mu \mathrm{g}$ of each protein. The R167A PfPdx1 protein had reduced PLP-producing capability and in the presence of only R5P the formation of $\mathrm{I}_{320}$ was diminished compared to WT PfPdx1.

Figure 4. E4P analogues and their effect on PfPdx1. (A) $4 \mathrm{PEHz}$ docked into $P b P d x 1$. Poses of 4PEHz were scored using LigScore1 and resulted in a value of 3.73, compared to 4.32 for the R5P control substrate. The hydrazide group of $4 \mathrm{PEHz}$ was orietated approxiately parralel to R150 in close proximity to D108, were both ketone and the hydroxyl substituents of 4PEHz hydrogen bonded with K84 and D27. (B) Representative UV-visible absorbance spectra of PfPdx1 with increasing concentrations of $4 \mathrm{PEHz}$ which resulted in decreased formation of PLP visible at $414 \mathrm{~nm}$. Formation of a $320 \mathrm{~nm}$ absorbing species was observed similar to E4P. Results represent single observations from independent triplicate experiments. (C) Efficacy of 4PEHz and EHz on purified PfPdx 1 with $4 \mathrm{PEHz}$ calculated to have an $\mathrm{IC}_{50}$ of $43 \pm 8 \mu \mathrm{M}$ and $\mathrm{EHz}$ an $\mathrm{IC}_{50}$ of $0.9 \pm 0.2 \mathrm{mM}$. (D) Effect of $4 \mathrm{PEHz}$ on the PfPdx 1 activity contained in the PLP synthase. Reaction conditions included $20 \mathrm{mM}$ glutamine, $0.5 \mathrm{mM}$ R5P 
and G3P, with $100 \mu \mathrm{g}$ of both PfPdx1 and PfPdx2. 4PEHz had an $\mathrm{IC}_{50}$ of $16 \pm 4 \mu \mathrm{M}$. Values in $\mathrm{C}$ and $\mathrm{D}$ represent data from three independent experiments performed in duplicate compared to an uninhibited control, in which error bars indicate the SEM, together with the $95 \%$ confidence interval in dotted lines.

Figure 5. Efficacy of $4 \mathrm{PEHz}$ on the proliferation of intra-erythrocytic $P$. falciparum parasites. (A) Parasite proliferation was assessed by monitoring the $\left[{ }^{3} \mathrm{H}\right]$-hypoxanthine incorporation. After $48 \mathrm{~h}$ parasite proliferation was reported as the percentage of $\left[{ }^{3} \mathrm{H}\right]-$ hypoxanthine incorporation compared to that for uninhibited control parasites. The $\mathrm{IC}_{50}$ for 4PEHz was $10.4 \pm 1.2 \mu \mathrm{M}$, compared to $138 \pm 9 \mu \mathrm{M}$ for EHz. Values represent data from more than three independent experiments performed in triplicate, were error bars represent the SEM together with the $95 \%$ confidence interval in dotted lines. (B) The growth of PfPdx1/PfPdx2-complemented and mock-control parasites was determined in the presence of $1 \mu \mathrm{M} 4 \mathrm{PEHz}$. The parasitemia (\% infected erythrocytes per 1000 erythrocytes) of the treated parasites was compared to untreated parasites and expressed as a percentage growth relative to the untreated control. Results represent average values from two independent experiments performed in triplicate, of which error bars indicate the SEM. Comparisons between treated and untreated parasites were made using an unpaired two-tailed Student's t-test in which ** represents statistical significance $(\mathrm{p}<0.05)$. The mock parasites were significantly affected after $48 \mathrm{~h}$, and had diminished growth over the $168 \mathrm{~h}$ period. Parasites complemented with PfPdx1 and PfPdx2 were not significantly affected by $4 \mathrm{PEHz}$ treatment, suggesting that $4 \mathrm{PEHz}$ affects PLP-related processes within the parasites, and complementation of PLP biosynthesis protects these parasites from the PfPdx1 inhibitor.

Figure 6. Schematic illustration of the formation of PLP from R5P, G3P and Lglutamine, as adapted from Hanes et al [10]. Initial binding of R5P in Pdx1 entails imine formation of the $\mathrm{C} 1$ of R5P with K83. Following binding, R5P undergoes isomerization, imine formation with ammonia, as well as a $\mathrm{C} 1$ to $\mathrm{C} 5$ lysine migration. Loss of phosphate results in the formation of $\mathrm{I}_{320}$, followed by imine formation with G3P, which leads to ring closure and formation of PLP. E4P and 4PEHz both inhibit PfPdx1 and share polyhydroxyl stereochemical arrangement and contain hydrolyzable phosphate groups similar to R5P. E4P contains a $\mathrm{C} 1$ aldehyde group, which is proposed to functionally mimic similar groups in R5P, and could facilitate entry into the PfPdx1 R5P-active site. 4PEHz, with a terminal hydrazide group, may similarly enter the active site, and interfere with PLP formation. 
Supplementary Figure 1: Detection of PLP is not affected by E4P. Standard curves of PLP, containing concentrations ranging from $10 \mu \mathrm{M}$ to $200 \mu \mathrm{M}$ PLP with no E4P (A), or containing $1 \mathrm{mM}(50 \mathrm{nmol}) \mathrm{E} 4 \mathrm{P}(\mathbf{B})$, in Tris- $\mathrm{HCl}$ buffer conditions including $0.5 \mathrm{mM}$ R5P and $\mathrm{G} 3 \mathrm{P}$ with $20 \mathrm{mM} \mathrm{NH}_{2} \mathrm{Cl}$. Reactions were incubated at $37{ }^{\circ} \mathrm{C}$ for $1.5 \mathrm{~h}$. Statistical analyses of the linear regression slope and intercepts revealed no significant differences in the slopes of the two different regression lines $(\mathrm{P}=0.975)$. Similarly, ANCOVA showed no significant difference $(\mathrm{P}=0.401)$ between the two regression lines. Results represent data from four independent experiments performed in triplicate. This verifies that molar excess concentrations of E4P did not affect detection of the PLP-Schiff base formed at $414 \mathrm{~nm}$.

Supplementary Figure 2: Effect of 4PEHz on mock and PfPdx1/PfPdx2 over-expressing transgenic parasites. Mock control cells (A) and PfPdx1/PfPdx2 over-expressing cells (B) were treated with $1 \mu \mathrm{M} 4 \mathrm{PEHz}$ in continuous culture for seven days. The cumulative parasitemia was calculated from the observed parasitemia taking the dilution factor into account. The data represents average values from two independent experiments performed in triplicate, in which error bars indicate the SEM. Statistical analyses using GraphPad Prism tested a null hypothesis that the lines were the same with a $99 \%$ confidence interval $(P=0.01$, $\mathrm{F}=0.01)$. Mock-UT and Mock-T $(\mathrm{B})$ had significantly different slopes $(P<0.0001)$. Whereas $P f \mathrm{Pdx} 1 / P f \mathrm{Pdx} 2-\mathrm{UT}$ and $P f \mathrm{Pdx} 1 / P f \mathrm{Pdx} 2-\mathrm{T}(\mathrm{C})$ did not have significantly different slopes $(P=$ 0.0138). Similarly ANCOVA analyses comparing MOCK-UT with MOCK-T revealed that the lines differed significantly (F-ratio probability $<0.0001$ ), whereas Pdx1/2-UT did not significantly differ from PfPdx1/PfPdx2-T (F-ratio probability $=0.0986)$.

Supplementary figure 3: Composition of parasite stages in mock control parasites during long-term exposure of 4PEHz. The parasite life-stage composition (percentage of rings, trophozoites or schizonts) of untreated (A) and treated (B) P. falciparum mock cells was determined microscopically during long term growth assays. Results represent average values from two independent experiments performed in triplicate, of which error bars indicate the SEM. Comparisons between treated and untreated mock parasites were made using an unpaired two-tailed Student's t-test in which ${ }^{* *}$ represents statistical significance $(p<0.05)$. The treated mock parasites had a significantly different ring and trophozoite life-stage composition compared to the untreated controls parasites at 120 hours while all other time points did not reveal significant differences in the life-stage compositions.

Supplementary figure 4: Composition of parasite stages in PfPdx1/PfPdx2complemented parasites during long-term exposure of 4PEHz. The parasite life-stage composition (percentage of rings, trophozoites or schizonts) of untreated (A) and treated (B) $P$. falciparum $P f \mathrm{Pdx} 1 / P f \mathrm{Pdx} 2$-overexpressing parasites was determined microscopically during long term growth assays. Results represent average values from two independent experiments performed in triplicate, of which error bars indicate the SEM. Comparisons between treated and untreated mock parasites were made using an unpaired two-tailed Student's t-test in which $* *$ represents statistical significance $(\mathrm{p}<0.05)$. There were no significant differences in the life-stage compositions between untreated and treated PfPdx1/PfPdx2 parasites at any of the time points. This confirmed that complementation of PLP biosynthesis protected the parasites from the effects of $4 \mathrm{PEHz}$, and the parasites grew normally compared to untreated cells. 
A

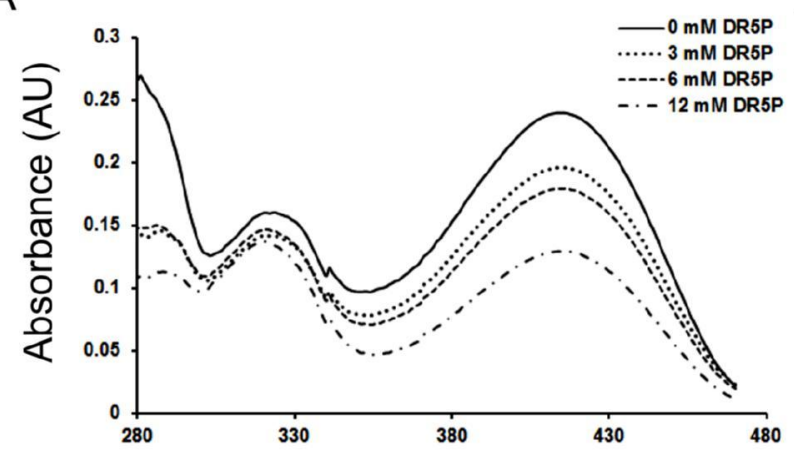

B

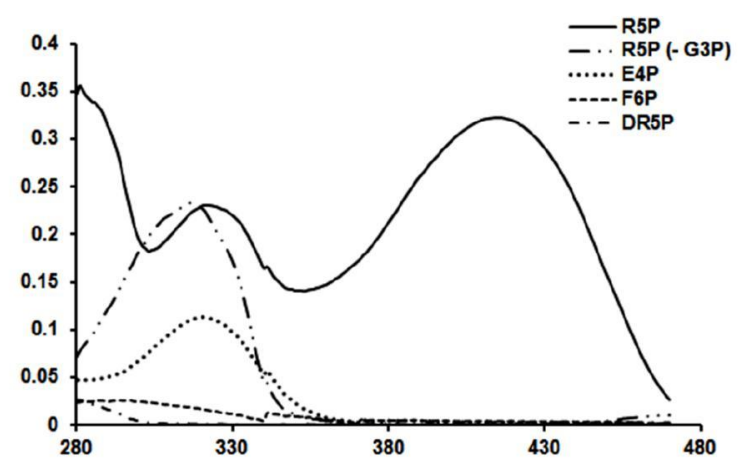

Wavelength $(\mathrm{nm})$

C

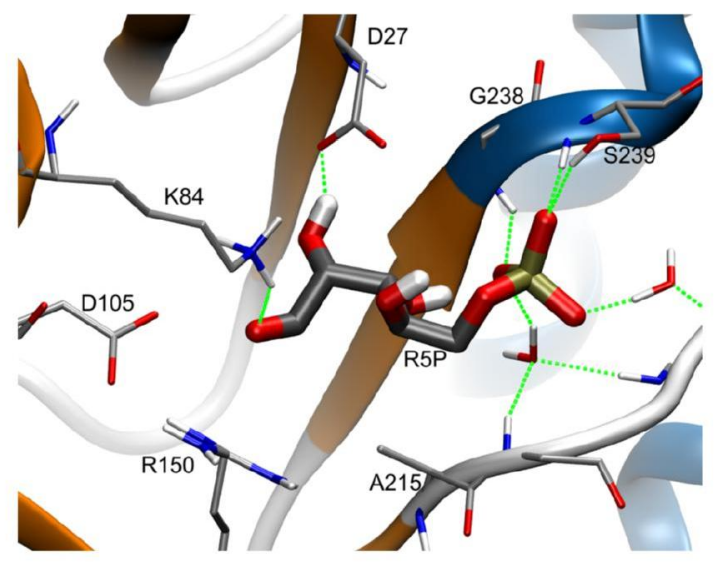

D

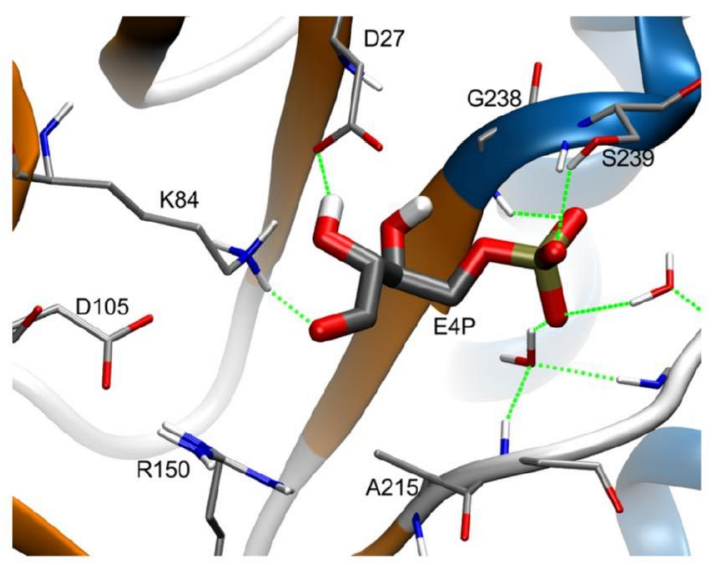




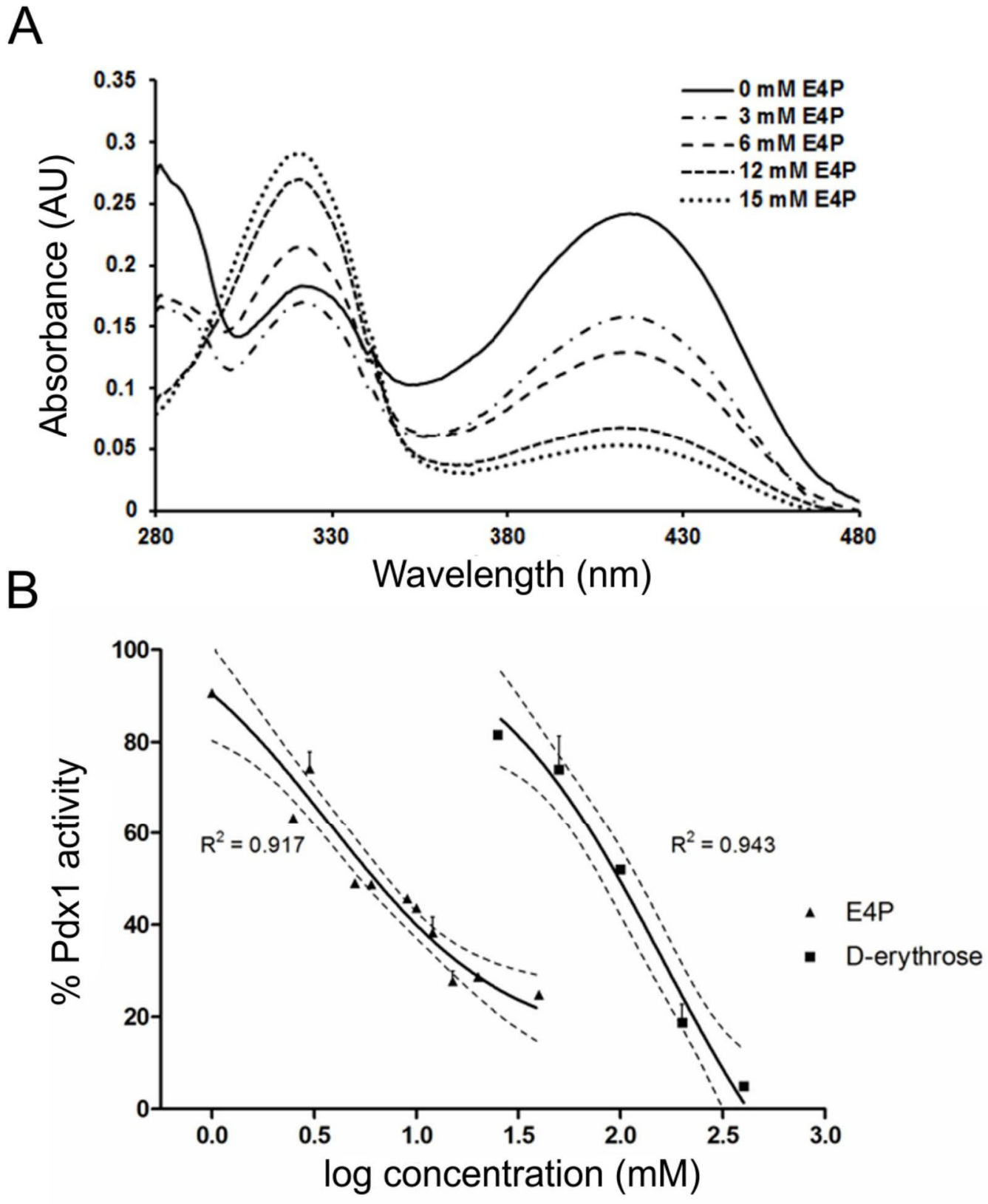


A

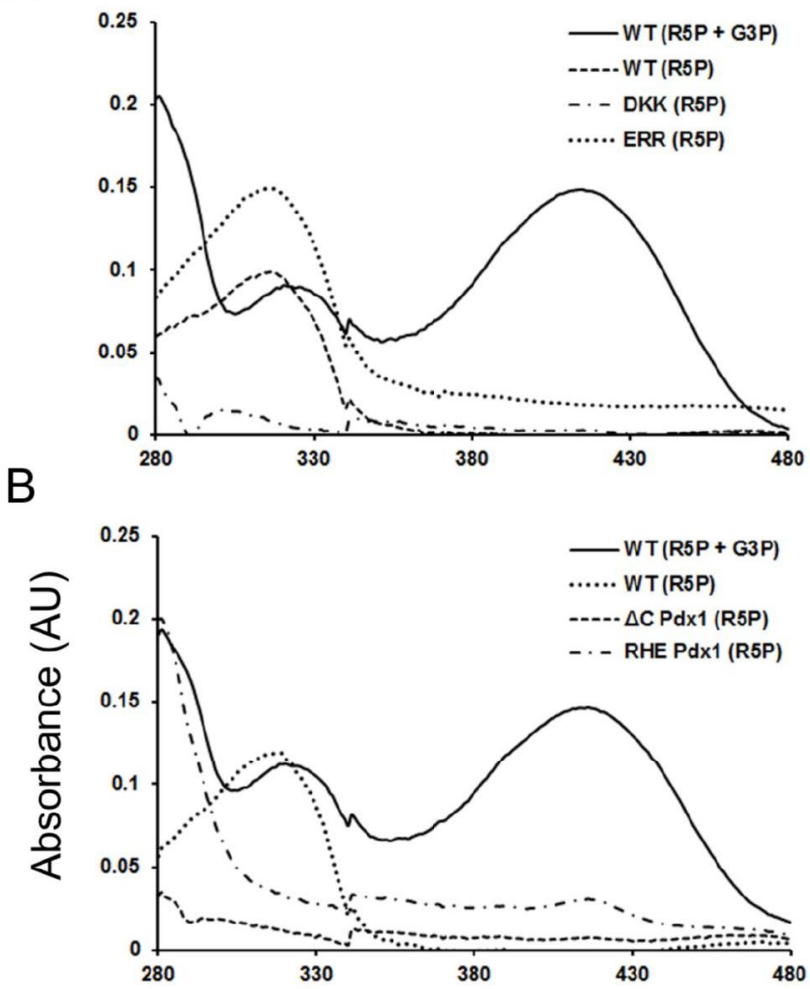

C

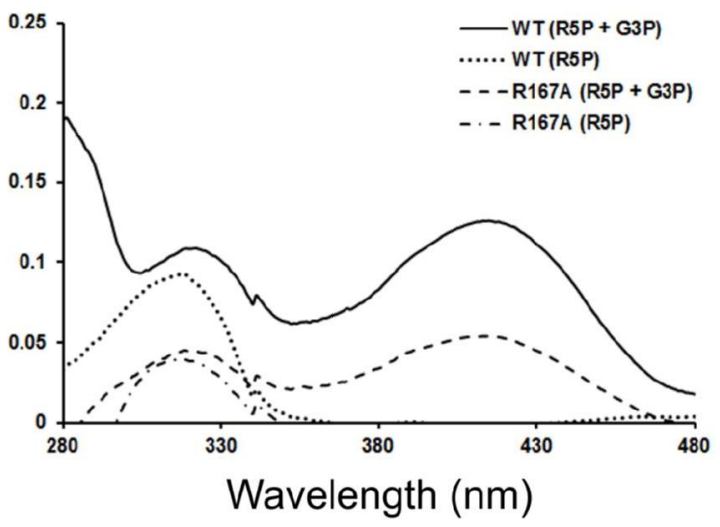


A

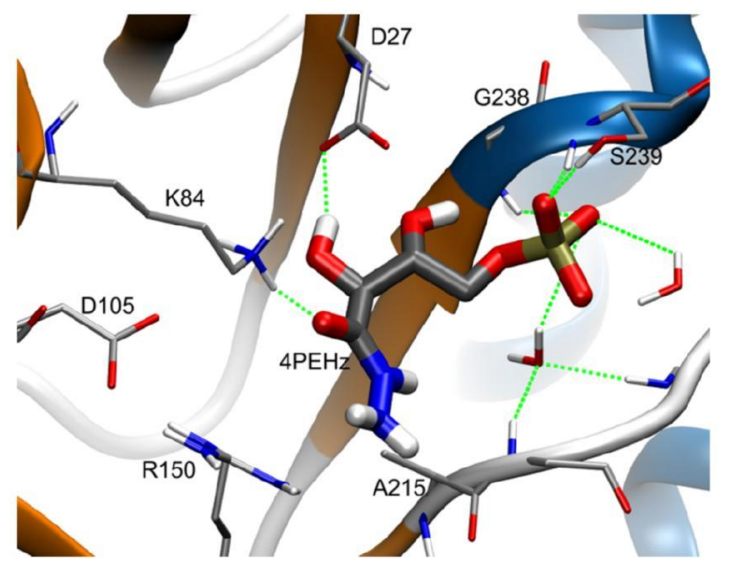

C

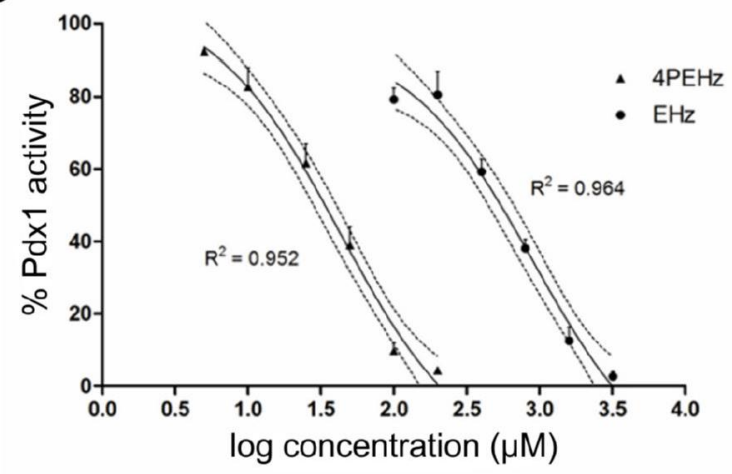

B

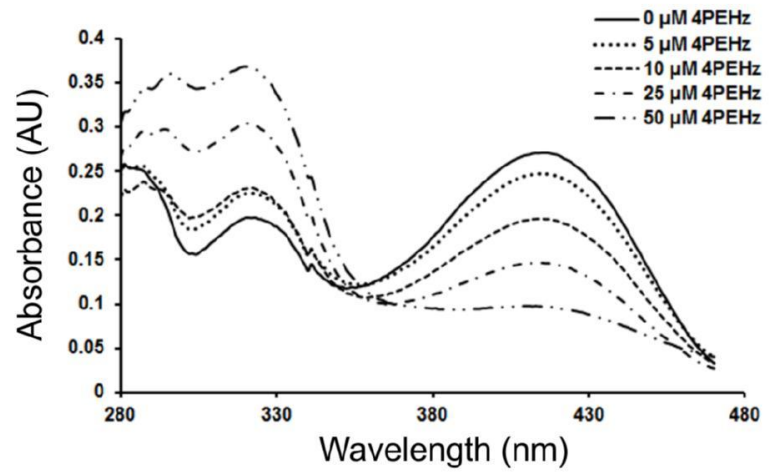

D

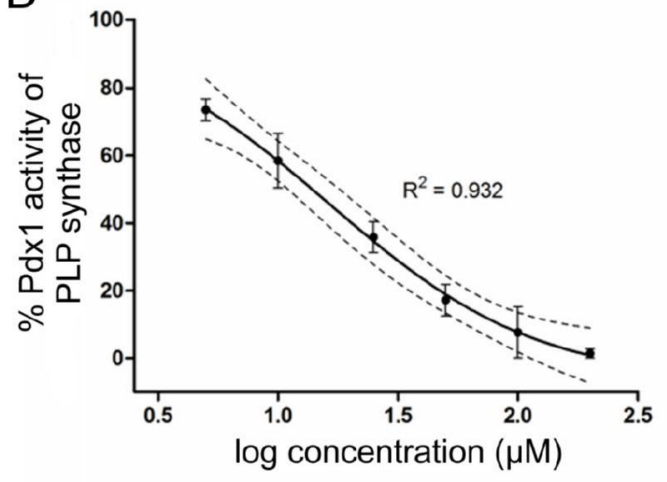


A
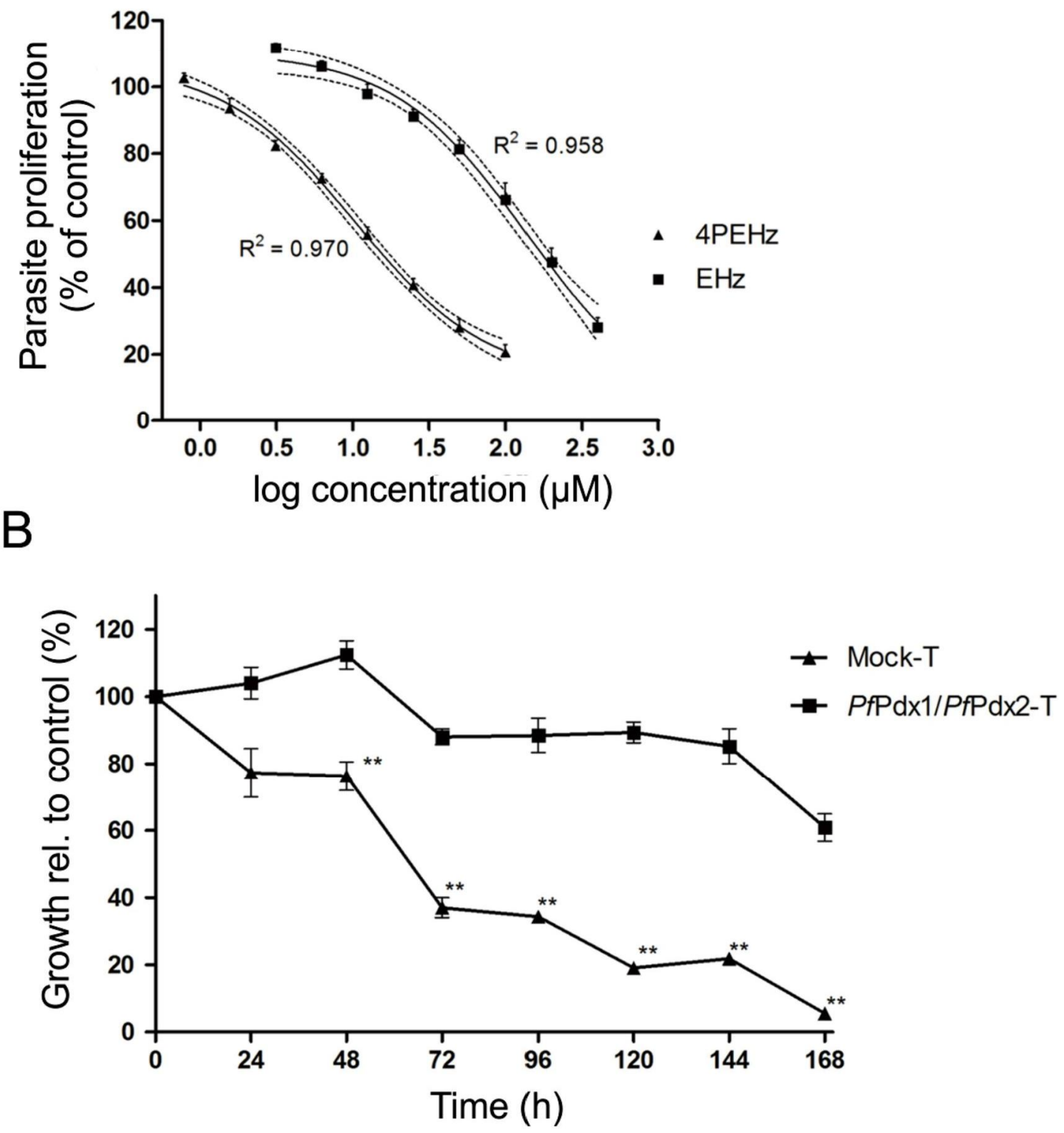

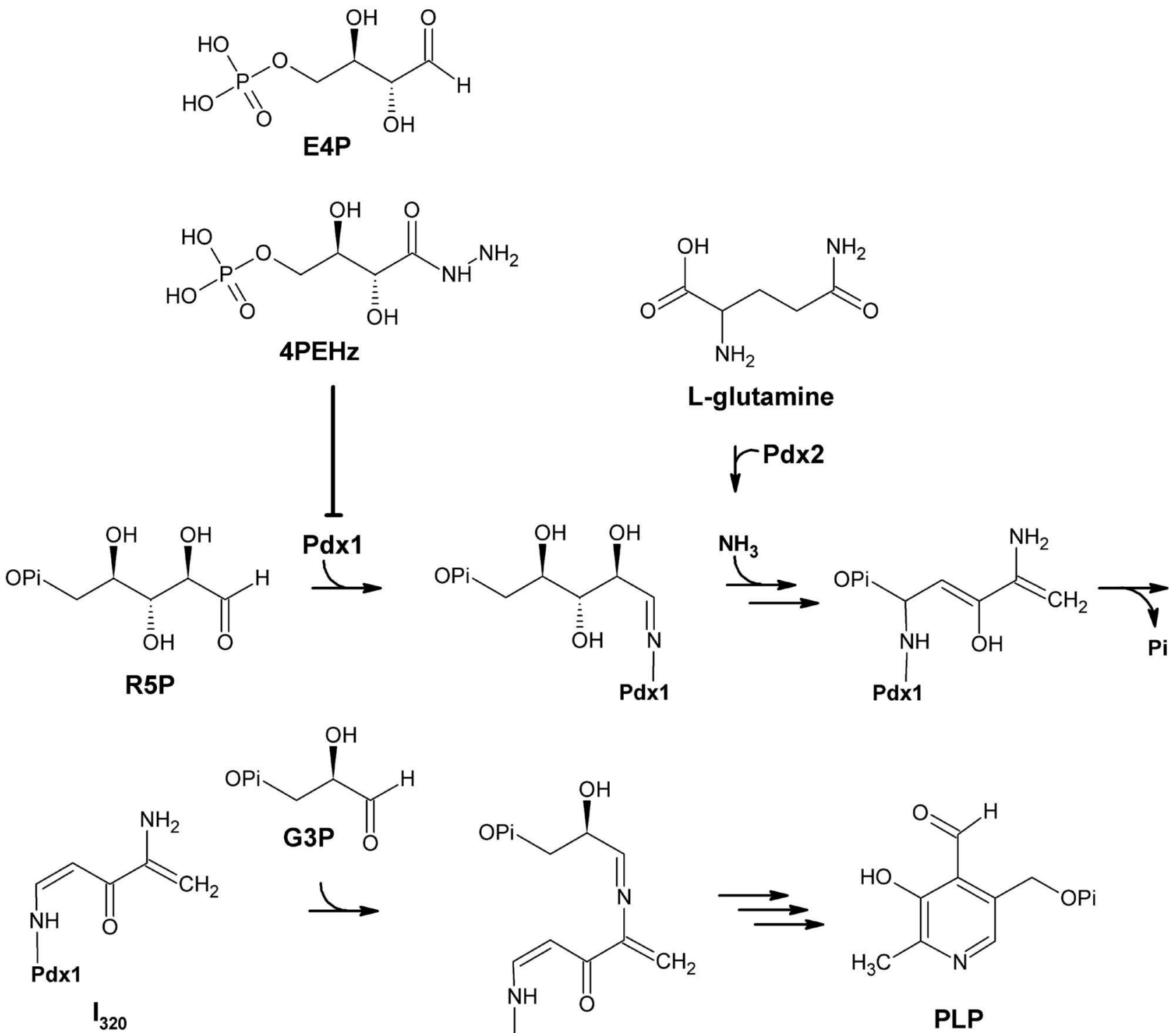

\section{L-glutamine}

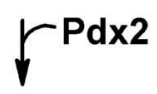<smiles>NC(=O)CCC(N)C(=O)O</smiles>

(2)

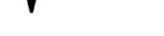

